\title{
Breaking the misconception of a dry and lifeless semiarid region: the diversity and distribution of aquatic flora in wetlands of the Brazilian Northeast
}

\author{
Lígia Queiroz Matias ${ }^{1 *}$ (D), Felipe Martins Guedes ${ }^{2}$ (D), Hugo Pereira do Nascimento ${ }^{1}$ (D) and Júlia Caram Sfair ${ }^{1}$ (D)
}

Received: May 19, 2020

Accepted: November 19, 2020

\begin{abstract}
The semiarid region of northeastern Brazil possesses a set of wetlands characterized by hydrographic basins with deficient drainage networks, a few large and permanent lotic systems and several permanent and temporary lagoons. Aquatic plants are widely distributed in these wetlands and the present study aims to determine if those of Ceará state have similar species compositions and differences in species richness. We hypothesized that lentic ecosystems would have more species and different growth forms of aquatic angiosperms than lotic ecosystems. A total of 1619 records of aquatic angiosperms in 43 wetland areas were analysed. The most representative families were Cyperaceae, Poaceae, Fabaceae, Alismataceae, Malvaceae, Nymphaeaceae and Pontederiaceae. Most of the species are helophytes and bottom-rooted emergent hydrophytes. Permanent lentic ecosystems had the highest number of exclusive species (27.85 \%), followed by temporary lentic ecosystems (20.54\%). Contrary to our hypothesis, the different aquatic ecosystems were found to possess distinct species compositions and different proportions of growth forms, and all wetland types contributed to the macrophyte richness of the study area, although they differ in species richness. Therefore, conservation plans for the native aquatic macrophyte biota should include all wetland ecosystems in the semiarid state of Ceará.
\end{abstract}

Keywords: biodiversity, floristic richness, hydrophytes, macrophytes, seasonal aquatic ecosystems

\section{Introduction}

Arid regions are traditionally perceived as relatively simple ecosystems, with low species diversity (McNeely 2003). However, the conclusions about the patterns of diversity in these regions can differ widely depending on the taxon analysed and the peculiarities of the geographical areas (MacKay 1991). If we consider that in temporary pools, the spatial structure of the aquatic plant community changes significantly during the rainy season according to the stage of flooding (Ferreira et al. 2015), would the diversity of aquatic plants in the semiarid region be differentiated between the lentic and lotic systems?

Wetlands in semiarid zones of northeastern Brazil are influenced by climatic seasonality and unpredictable flood pulses, which present multiannual frequency and low amplitude (Junk et al. 2014). A biogeographic delineation approach of South American freshwater ecosystems considered the extreme Northeast of Brazil as the "Ecoregion

1 Centro de Ciências, Departamento de Biologia, Universidade Federal do Ceará, 60440-900, Fortaleza, CE, Brazil

2 Programa de Pós-Graduação em Biologia Vegetal, Universidade Federal de Pernambuco, 50670-901, Recife, PE, Brazil

* Corresponding autor: lqmatias@ufc.br 
Northeastern Caatinga and Coastal drainages" (Abell et al. 2008), with a predominance of aquatic systems albeit of low water volume, but functional in terms of regulation of the regional hydrological regime and maintenance of biodiversity (Junk et al. 2014). Particularly in this ecoregion, Ceará state presents the largest flood area $(24,339.65 \mathrm{ha})$ and the largest number of temporary lagoons $(2,930)$ (Maltchick et al. 1999). Therefore, these temporary lagoons, together with the hydrographic basins with a deficient drainage network and the permanent lentic systems of coastal formations (Claudino-Sales \& Peulvast 2002), characterize the set of wetlands in the state.

Temporary lagoons have a cyclical nature, involving alternating rainy and dry seasons, which favours the succession of different species in processes of flooding and drought (Tabosa et al. 2012). In contrast, the permanent lagoons, given the stability of the water column, have plant communities associated with the depth of the euphotic zone, as this is a determining factor for the extension of the habitats of macrophytes (Wetzel 2001). Furthermore, in artificial ecosystems like weirs, aquatic plant assemblages are more associated with nutrient concentrations than with water depth (Paiva et al. 2014).

On the other hand, lotic systems in the Brazilian semiarid region are characterized by flash floods during the rainy season that can vary according to rainfall, i.e., the water can flow for weeks in small streams or months in larger rivers during the 'wet phase' (Maltchick \& Medeiros 2006). During the dry season or "drying phase", water flow ceases, leading to the formation of strings of disconnected temporary pools along the riverbed where the aquatic biota survives (Medeiros \& Maltchick 1999). In addition, due to the absence of the river-floodplain system, only the main river channel keeps the temporary pools in the dry season, resulting in less habitat availability for aquatic organisms (Maltchick \& Medeiros 2006).

Along the coast of Ceará, permanent and temporary aquatic systems originate in interdune and deflated dune areas, as a result of flooding during the rainfall period or the rise of the water column in less permeable soils, and are located mainly between old Quaternary dunes and Tertiary Formations (also known as "Formação Barreiras" [ClaudinoSales \& Peuvast 2002]). In addition to these, permanent lentic ecosystems originate from the barrage of rivers in the vicinity of their mouths due to the accumulation of sand carried by the wind (forming the "lagamares"), or by abandoned meanders and marginal lagoons located along corridors of the main rivers that reach the coast (Silva et al. 2007).

Aquatic plants occur both in coastal environments (Matias et al. 2003; Moro et al. 2014) and in temporary and permanent aquatic systems, natural or artificial, located in the semiarid region (Paiva et al. 2014; Albuquerque et al. 2020). In shallow lagoons, plant communities occupy the entire water column, forming strata of submerged hydrophytes, overlaid by bottom-rooted emergents with floating leaves and/or stems, and by bottom-rooted emergents above the water surface (Tabosa et al. 2012). In deep permanent reservoirs and lagoons, the communities occur at shallower depths and periodically flooded banks (Matias et al. 2003; Paiva et al. 2014). In lotic ecosystems, species richness is lower in systems with deficient drainage, and communities are influenced by the flood intensity (Pedro et al. 2006).

Growth forms vary according to the stability of the water column, with the presence of submerged forms in permanent lentic systems being more common (Matias et al. 2003; Moro et al. 2014; Paiva et al. 2014) while the bottom-rooted emergents and bottom-rooted emergents with floating leaves and/or stems share the water surface in temporary lentic systems (Tabosa et al. 2012).

On the other hand, the dynamics of the water column in permanent lotic systems constitute a strong environmental filter to colonization by aquatic plants, which depend on marginal areas, such as an underwater banks or places with water between the spit and the shore, which are protected from strong turbulence (Sculthorpe 1967). The bottomrooted emergent and bottom-rooted submerged forms occur in these marginal habitats (Koehler \& Bove 2004), whilst haptophytes are restricted to habitats with accentuated unevenness (Silva et al. 2015). And, in temporary lotic systems, the occurrence of two phases of hydrological disturbance (flooding and drought) exert a strong influence so that angiosperms with bottom-rooted submerged or freefloating forms are observed in the areas of river resurgences or permanent river puddles (Maltchick \& Medeiros 2006; Maltchick \& Bianchini 2006).

Considering that aquatic plants are widely distributed in wetlands, the present study analyses if aquatic systems of Ceará state tend to show similarity in species composition and differences in species richness and growth forms. We hypothesize I) that lentic ecosystems, natural or artificial, will exceed lotic ecosystems in the number of species (richness). However, as rivers present drainage deficiency and discontinuity (having stability of the water column for only a few months), we expect that II) lentic and lotic ecosystems present similarity in species composition and differences in the proportions of growth forms.

\section{Materials and methods}

\section{Data source}

All records of angiosperms (number of species in each area) from wetlands of Ceará state were obtained through systematic inventories carried out in the state, collections were performed during the wet seasons from 2000 to 2020 and are published elsewhere and we also included data (number of species) from Iguatu wetlands from unpublished studies (Tab. 1). In the case of these unpublished studies, all species were sampled in Iguatu wetlands along the shoreline 
of the lagoons using three transects $50 \mathrm{~m}$ apart, from the margin to the furthermost area of the macrophyte stand, close to the limnetic zone. Plants were sampled in a belt transect ca. $1 \mathrm{~m}$ wide using traditional plant sampling tools (scissors, shovels and hoes), given that the depth of the water column (less than $1 \mathrm{~m}$ ) did not require any other apparatus.

Nomenclatural data were updated based on IPNI (http:// www.ipni.org/), Flora do Brasil 2020 (http://reflora.jbrj.gov. br), Tropicos (http://tropicos.org) and The Plant List (http:// theplantlist.org). Identifications made by specialists were considered and others had their identifications updated based on the specific literature for each family. The new records and origin status (native or exotic) were based on Flora do Brasil 2020 (2020), in order to highlight the main invasive species, we annotated this information after each scientific name in the species list (Moro et al. 2012). Growth forms were classified following Cook (1990) as the following: (1) Hydrophytes: bottom-rooted submerged (RS), bottom-rooted emergent (RE), bottom-rooted emergent with floating leaves and/or stems (RLF), free-swimming submerged (FS), free-floating emergent (FE), and (2) Helophytes (Hel).

For the similarity analysis, the following 43 wetland habitats were selected: 9 Permanent Lentic (PLE), 11 Temporary Lentic (TLE), 8 Permanent Lotic (PLO), 7 Temporary Lotic (TLO) and 8 Artificial Lentic (ALE) ecosystems (Fig. 1, Tab. S1 in supplementary material).

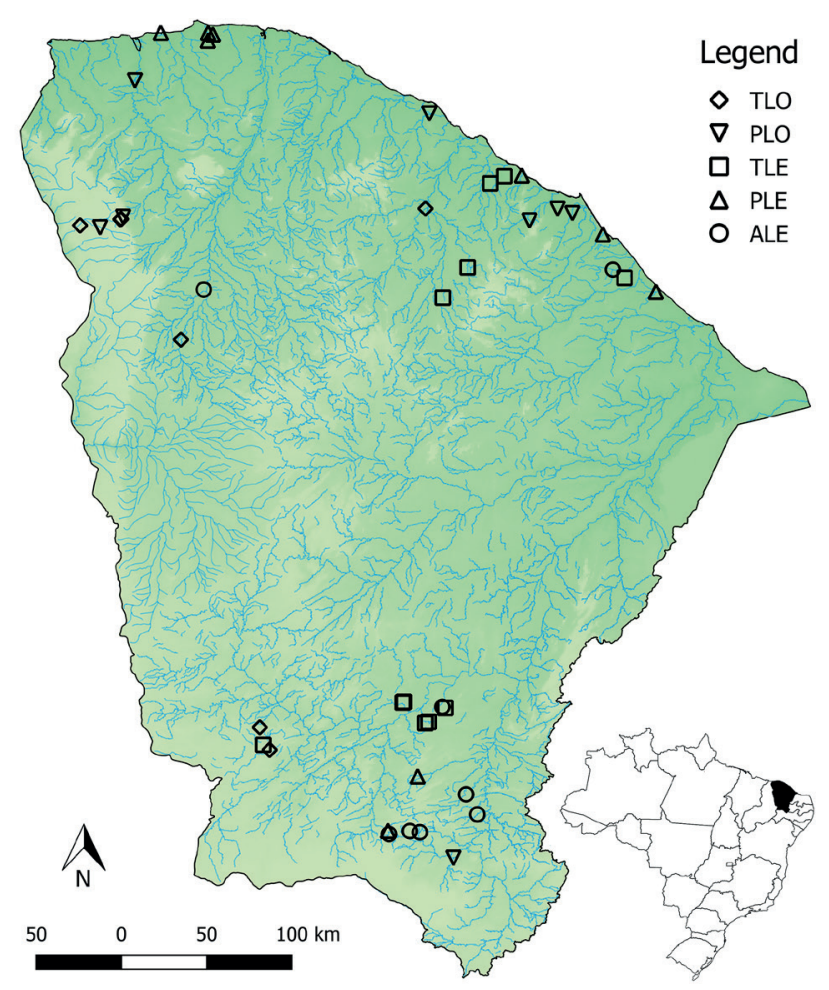

Figure 1. Map of the localization of the 43 selected wetland areas. ALE $=$ Artificial Lentic ecosystem $;$ PLE $=$ Permanent Lentic ecosystem; PLO = Permanent Lotic ecosystem; TLE = Temporary Lentic ecosystem; TLO = Temporary Lotic ecosystem.

\section{Statistical analyses}

To compare the richness among the five different ecosystems (PLE, TLE, PLO, TLO and ALE) we used the extrapolation approach based on the Hill number with $\mathrm{q}$ $=0$ (Chao et al. 2014), using the iNEXT package (Hsieh et al. 2013) in R software (R Development Core Team 2020). We performed 1000 randomizations and calculated the confidence interval at $95 \%$.

We calculated the distance of species composition among the 43 ponds and rivers with the Jaccard dissimilarity index. The distance matrix was related to their features (lentic or lotic and permanent, temporary or artificial) with a PERMANOVA approach, which is a multivariate analysis of variance for dissimilarity data with permutations (Anderson 2001; McArdle \& Anderson 2001). PERMANOVA does not have the assumption of normal distribution, being characterized as a non-parametric analysis more powerful than the analysis of similarity (ANOSIM) and the Mantel test in detecting differences in real communities (Anderson \& Walsh 2013). PERMANOVA also assumes independent observations, it can use categorical or continuous independent variables, and it uses a permutation test (Anderson 2001). We used the "adonis" function with 10,000 replications, followed by the "betadisper" function in the "vegan" package (Oksanen et al. 2019) in R software (R Development Core Team 2020).

To understand the proportion of growth forms, considering the interaction of lotic/lentic and temporary/ artificial/permanent ecosystems, we calculated the ScheirerRay-Hare test, which is a non-parametric test analogous to the two-way ANOVA when their assumptions are not met (Sokal \& Rohlf 1995). We used the proportion of growth forms because the number of sampled units of Permanent Lentic (9), Temporary Lentic (11), Permanent Lotic (8), Temporary Lotic (7) and Artificial Lentic (8) were different and, thus, not comparable concerning the absolute number of species in each growth form.

Additionally, we performed the post-hoc pairwise Dunn test (Sokal \& Rohlf 1995). The Scheirer-Ray-Hare test was calculated with the "rcompanion" package (Mangiafico 2019) and the Dunn test with the "FSA" package (Ogle et al. 2020), both in R software (R Development Core Team 2020).

\section{Results}

Our sampling of aquatic angiosperms is represented by 44 families, 108 genera and 219 species (Tab. 1). The most representative families were Cyperaceae ( $23 \%$ ), Fabaceae (10\%), Poaceae (9\%), Alismataceae (5\%), followed by Araceae, Nymphaeaceae and Pontederiaceae (4\% each) (Fig. 2A). Most of the species are helophytes (58\%) and bottom-rooted emergent hydrophytes (22\%) (Fig. 2B). Of the species in this study, 21 are new records for Ceará state (Flora do Brasil 2020 2020), most representatives of Poaceae (7 spp.). 
The greatest values for species richness were found in permanent (162 spp.) and temporary lentic (107 spp.) ecosystems, followed by artificial lentic ecosystems (86 spp.). Temporary (32 spp.) and permanent lotic (51 spp.) ecosystems presented the lowest richness (Fig. 3). Six species occurred in all five ecosystems: Echinodorus subalatus, Pistia stratiotes, Neptunia plena, Nymphaea amazonum, Ludwigia helminthorrhiza and Ludwigia leptocarpa. Permanent lentic ecosystems showed the largest number of exclusive species ( 61 or $27.85 \%$ ), followed by temporary lentic (45 or $20.54 \%$ ), artificial lentic (14 or $6.39 \%$ ), permanent lotic (12 or $5.47 \%$ ) and temporary lotic ( 8 or $3.65 \%$ ). Most of the species (123 or $56.16 \%$ ) occurred exclusively in nonartificial lentic ecosystems.
The five ecosystems have different species compositions $\left(\mathrm{F}=1.25 ; \mathrm{R}^{2}=0.03 ; \mathrm{P}=0.03\right)$, but no significant dispersion $\left(\mathrm{F}_{4.38}=0.87 ; \mathrm{P}=0.48\right)$, indicating that the difference among the five ecosystems is greater than the difference within the ecosystems (Fig. 4; Tab. 2). Temporary aquatic systems tend to have higher proportions of free-swimming submerged and bottom-rooted emergents with floating leaves and/or stems (Fig. 5A and B, Tab. S2 in supplementary material), whereas lentic ecosystems tend to have higher proportions of bottom-rooted submerged forms than lotic ecosystems (Fig 5C, Tab. S2 in supplementary material). On the other hand, helophytes tend to show different proportions among the different ecosystems (Fig. 5D, Tab. S2 in supplementary material).

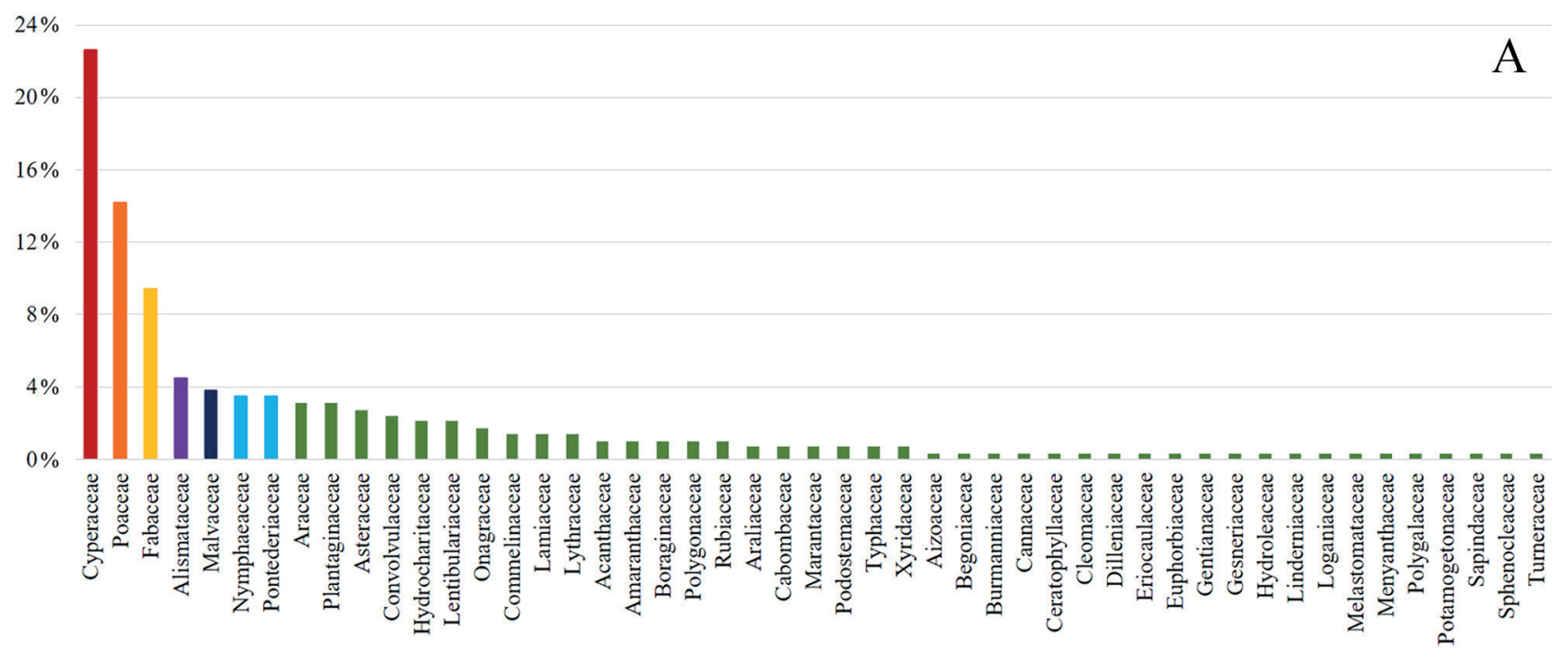

$100 \%$

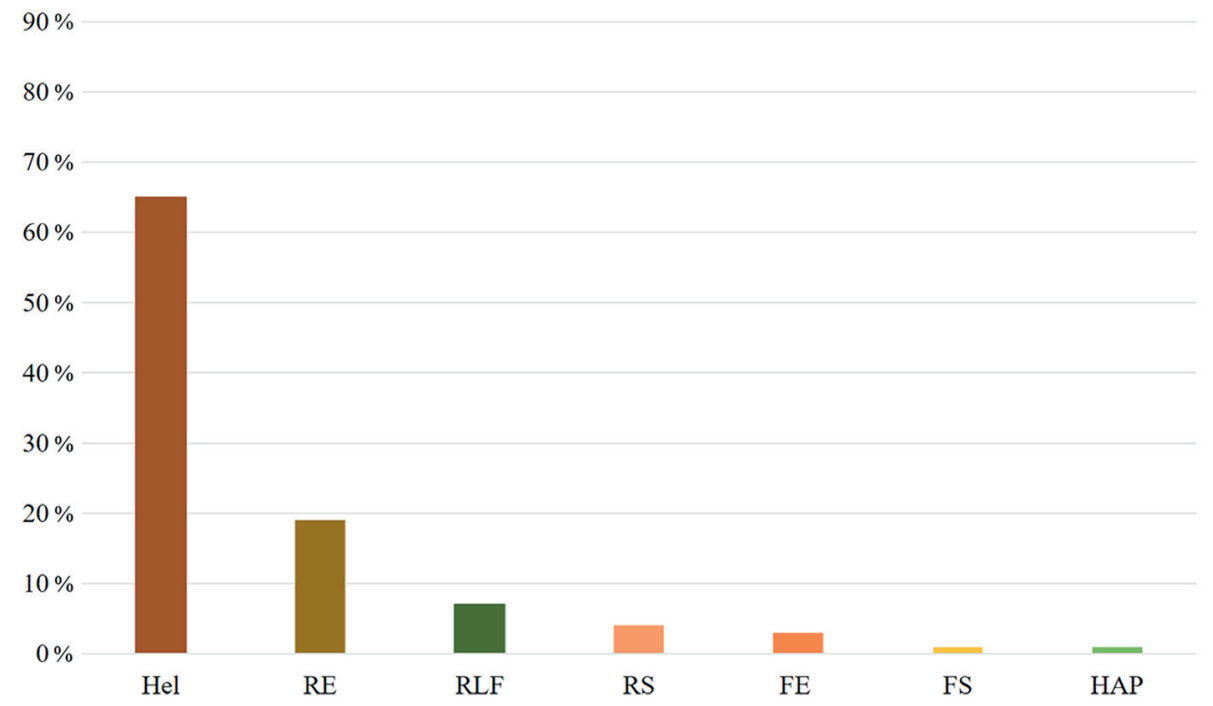

Figure 2. A. Percentage of species richness per family in the study areas. B. Percentage of growth forms (Cook 1990) of aquatic angiosperms in the study areas. (Hel) Helophytes; (RE) Bottom-rooted emergent; (RLF) Bottom-rooted emergent with floating leaves and/or stems; (RS) Bottom-rooted submerged; (FE) Free-floating emergent; (FS) Free-swimming submerged; (HAP) Haptophytes. 
Lígia Queiroz Matias, Felipe Martins Guedes,

Hugo Pereira do Nascimento and Júlia Caram Sfair

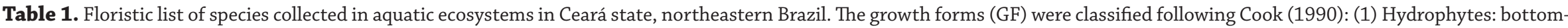

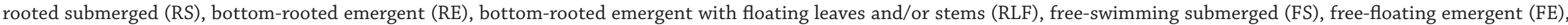

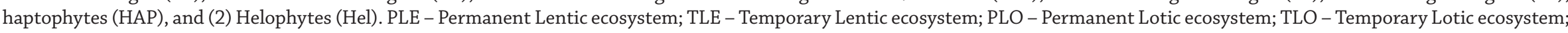
ALE - Artificial Lentic ecosystem. $\left(^{*}\right)$ New record for Ceará state.

\begin{tabular}{|c|c|c|c|c|c|c|c|c|}
\hline Family & Species & Occurence indication & GF & PLE & TLE & PLO & TLO & ALE \\
\hline \multirow{2}{*}{ Acanthaceae } & Hygrophila guianensis Nees ex Benth. ${ }^{(*)}$ & Guedes \& Nascimento 2016 & Hel & 0 & 0 & 1 & 0 & 0 \\
\hline & Justicia laevilinguis (Nees) Lindau & Normando 2011 & Hel & 0 & 1 & 0 & 0 & 0 \\
\hline Aizoaceae & Sesuvium portulacastrum (L.) L. & Matias \& Nunes 2001 & Hel & 1 & 1 & 0 & 0 & 0 \\
\hline \multirow{12}{*}{ Alismataceae } & Echinodorus glandulosus Rataj & Matias \& Sousa 2011 & $\mathrm{RE}$ & 0 & 1 & 1 & 1 & 0 \\
\hline & Echinodorus lanceolatus Rataj & Ibiapina-Santos 2016 & $\mathrm{RE}$ & 0 & 1 & 0 & 0 & 0 \\
\hline & Echinodorus palaefolius (Nees \& Mart.) J.F. Macbr. & Matias \& Sousa 2011 & $\mathrm{RE}$ & 0 & 1 & 0 & 0 & 0 \\
\hline & Echinodorus pubescens (Mart.) Seub. Ex Warm. & Ibiapina-Santos 2016 & $\mathrm{RE}$ & 0 & 1 & 0 & 0 & 0 \\
\hline & Echinodorus subalatus (Mart.) Griseb. & Tabosa et al. 2012; Moro et al. 2014; Ibiapina-Santos 2016; Albuquerque et al. 2020 & $\mathrm{RE}$ & 1 & 1 & 1 & 1 & 1 \\
\hline & Helanthium tenellum (Mart.) Britton & Matias et al. 2003 & $\mathrm{RE}$ & 1 & 0 & 0 & 0 & 1 \\
\hline & Hydrocleys martii Seub. & Tabosa et al. 2012; Ibiapina-Santos 2016; Albuquerque et al. 2020 & RLF & 1 & 1 & 0 & 0 & 0 \\
\hline & Hydrocleys nymphoides (Willd.) Buchenau & Ibiapina-Santos 2016 & RLF & 1 & 1 & 1 & 0 & 0 \\
\hline & Limnocharis flava (L.) Buchenau & Ibiapina-Santos 2016 & $\mathrm{RE}$ & 1 & 1 & 0 & 0 & 0 \\
\hline & Limnocharis laforesti Duchass. ex Griseb. & Ibiapina-Santos 2016 & $\mathrm{RE}$ & 0 & 1 & 0 & 0 & 1 \\
\hline & Sagittaria guayanensis Kunth. & Matias \& Sousa 2011 & RLF & 1 & 1 & 0 & 0 & 0 \\
\hline & Sagittaria planitiana G. Agostini & Matias \& Sousa 2011 & $\mathrm{RE}$ & 1 & 1 & 1 & 0 & 0 \\
\hline \multirow{3}{*}{ Amaranthaceae } & Alternanthera brasiliana (L.) Kuntze & Matias \& Nunes 2001 & Hel & 0 & 0 & 1 & 0 & 0 \\
\hline & Alternanthera tenella Colla & Ibiapina-Santos 2016 & Hel & 0 & 1 & 0 & 0 & 0 \\
\hline & Amaranthus spinosus L. $\left({ }^{*}\right)$ & Ibiapina-Santos 2016 & Hel & 0 & 1 & 0 & 0 & 0 \\
\hline \multirow{9}{*}{ Araceae } & Landoltia punctata (G. Mey.) Les \& D.J. Crawford & Andrade et al. 2013 & $\mathrm{FE}$ & 1 & 0 & 0 & 0 & 0 \\
\hline & Lemna aequinoctialis Welw. & Ibiapina-Santos 2016 & $\mathrm{FE}$ & 1 & 1 & 0 & 0 & 1 \\
\hline & Lemna minuta Kunth. & Ibiapina-Santos 2016 & $\mathrm{FE}$ & 0 & 1 & 0 & 0 & 0 \\
\hline & Montrichardia arborescens (L.) Schott ${ }^{(")}$ & Andrade et al. 2013 & $\mathrm{RE}$ & 1 & 0 & 0 & 0 & 0 \\
\hline & Montrichardia linifera (Arruda) Schott & Moro et al. 2014 & $\mathrm{RE}$ & 1 & 0 & 1 & 0 & 0 \\
\hline & Pistia stratiotes L. & Paiva et al. 2014; Ibiapina-Santos 2016; Albuquerque et al. 2020 & $\mathrm{FE}$ & 1 & 1 & 1 & 0 & 1 \\
\hline & Spirodela intermedia W. Koch & Andrade et al. 2013 & $\mathrm{FE}$ & 1 & 0 & 0 & 0 & 1 \\
\hline & Wolffia columbiana Karsten & Ibiapina-Santos 2016 & $\mathrm{FE}$ & 1 & 1 & 0 & 0 & 0 \\
\hline & Wolffiella welwitschii (Hegelm) Monod & Ibiapina-Santos 2016 & $\mathrm{FE}$ & 1 & 1 & 0 & 0 & 1 \\
\hline \multirow{2}{*}{ Araliaceae } & Hydrocotyle bonariensis Lam. ${ }^{(*)}$ & Moro et al. 2014 & Hel & 1 & 0 & 0 & 0 & 0 \\
\hline & Hydrocotyle verticillata Thunb. ${ }^{(")}$ & Guedes \& Nascimento 2016 & Hel & 1 & 0 & 0 & 0 & 0 \\
\hline \multirow{4}{*}{ Asteraceae } & Eclipta prostrata (L.) L. & Ibiapina-Santos 2016 & Hel & 1 & 1 & 1 & 0 & 1 \\
\hline & Erechtites hieracifolius (L.) Raf. ex DC. & Guedes \& Nascimento 2016 & Hel & 1 & 0 & 0 & 0 & 1 \\
\hline & Mikania micrantha Kunth & Guedes \& Nascimento 2016 & Hel & 1 & 0 & 0 & 0 & 0 \\
\hline & Spilanthes acmella (L.) L. (") & Guedes \& Nascimento 2016 & Hel & 0 & 1 & 0 & 0 & 0 \\
\hline Boraginaceae & Euploca polyphylla (Lehm.) J.I.M. Melo \& Semir & Guedes \& Nascimento 2016 & Hel & 1 & 1 & 0 & 0 & 0 \\
\hline Burmaniaceae & Burmannia capitata (Walter ex J.F. Gmel.) Mart. & Matias et al. 2003 & Hel & 0 & 1 & 0 & 0 & 0 \\
\hline
\end{tabular}


Table 1. Cont.

\begin{tabular}{|c|c|c|c|c|c|c|c|c|}
\hline Family & Species & Occurence indication & GF & PLE & TLE & PLO & TLO & ALE \\
\hline \multirow{2}{*}{ Cabombaceae } & Cabomba aquatica Aubl. & Ibiapina-Santos 2016 & RS & 1 & 1 & 1 & 0 & 0 \\
\hline & Cabomba haynesii Wiersema & Ibiapina-Santos 2016 & RS & 0 & 1 & 0 & 0 & 1 \\
\hline Cannaceae & Canna glauca L. & Ibiapina-Santos 2016 & $\mathrm{RE}$ & 1 & 1 & 0 & 0 & 0 \\
\hline Ceratophyllaceae & Ceratophyllum demersum L. & Moro et al. 2014; Paiva et al. 2014; Ibiapina-Santos 2016 & FS & 1 & 1 & 1 & 0 & 1 \\
\hline Cleomaceae & Tarenaya spinosa (Jacq.) Raf. & Paiva et al. 2014; Ibiapina-Santos 2016 & Hel & 1 & 1 & 1 & 1 & 1 \\
\hline \multirow{4}{*}{ Commelinaceae } & Callisia filiformis (M. Martens \& Galeotti) D.R. Hunt & Tabosa et al. 2012 & Hel & 0 & 1 & 0 & 0 & 0 \\
\hline & Commelina difusa Burm.f. & Matias \& Nunes 2001 & Hel & 0 & 1 & 1 & 0 & 0 \\
\hline & Dichorisandra perforans C.B. Clarke & Guedes \& Nascimento 2016 & Hel & 0 & 0 & 1 & 0 & 0 \\
\hline & Tripogandra glandulosa (Seub.) Rohweder(*) & Guedes \& Nascimento 2016 & Hel & 0 & 0 & 1 & 0 & 0 \\
\hline \multirow{5}{*}{ Convolvulaceae } & Aniseia martinicensis var. ambigua Hallier $\mathrm{f}$. & Ibiapina-Santos 2016 & Hel & 0 & 1 & 0 & 0 & 0 \\
\hline & Camonea umbellata (L.) A.R. Simões \& Staples & Normando 2011 & Hel & 0 & 0 & 1 & 0 & 0 \\
\hline & Ipomoea asarifolia (Desr.) Roem. \& Schult. & Matias et al. 2003; Moro et al. 2014; Ibiapina-Santos 2016 & Hel & 1 & 1 & 0 & 0 & 1 \\
\hline & $\begin{array}{l}\text { Ipomoea carnea subsp. Fistulosa } \\
\text { (Mart. Ex Choisy) D. F. Austin }\end{array}$ & Ibiapina-Santos 2016 & Hel & 0 & 1 & 0 & 0 & 0 \\
\hline & Ipomoea nil (L.) Roth & Ibiapina-Santos 2016 & Hel & 0 & 1 & 0 & 0 & 0 \\
\hline \multirow{24}{*}{ Cyperaceae } & Bulbostylis capillaris (L.) C.B. Clarke & Matias \& Nunes 2001 & Hel & 1 & 1 & 0 & 0 & 0 \\
\hline & Cyperus aggregatus (Willd.) Endl. & Matias et al. 2003; Moro et al. 2014; Ibiapina-Santos 2016 & Hel & 1 & 1 & 0 & 0 & 1 \\
\hline & Cyperus amabilis Vahl & Matias et al. 2003 & Hel & 1 & 0 & 0 & 0 & 0 \\
\hline & Cyperus articulatus L. & Moro et al. 2014 & Hel & 1 & 1 & 0 & 0 & 0 \\
\hline & Cyperus blepharoleptos Steud. & Paiva et al. 2014; Ibiapina-Santos 2016 & Hel & 1 & 1 & 0 & 0 & 1 \\
\hline & Cyperus brevifolius (Rottb.) Endl. ex Hassk. & Moro et al. 2014 & $\mathrm{Hel}$ & 1 & 0 & 0 & 0 & 0 \\
\hline & Cyperus compressus $\mathrm{L}$. & Moro et al. 2014; Ibiapina-Santos 2016 & Hel & 1 & 1 & 0 & 0 & 0 \\
\hline & Cyperus digitatus Roxb. & Ibiapina-Santos 2016 & $\mathrm{Hel}$ & 1 & 1 & 0 & 0 & 1 \\
\hline & Cyperus esculentus $\mathrm{L}$. & Ibiapina-Santos 2016 & Hel & 1 & 1 & 1 & 0 & 1 \\
\hline & Cyperus fugax Liebm. & Guedes \& Nascimento 2016 & Hel & 1 & 0 & 0 & 0 & 0 \\
\hline & Cyperus iria L. & Ibiapina-Santos 2016 & Hel & 0 & 1 & 0 & 0 & 0 \\
\hline & Cyperus ligularis $\mathrm{L}$. & Matias et al. 2003; Moro et al. 2014; Ibiapina-Santos 2016 & Hel & 1 & 1 & 0 & 0 & 1 \\
\hline & Cyperus luzulae (L.) Retz. & Guedes \& Nascimento 2016 & Hel & 0 & 1 & 1 & 0 & 0 \\
\hline & Cyperus macrostachyos Lam. & Ibiapina-Santos 2016 & Hel & 1 & 1 & 1 & 0 & 1 \\
\hline & Cyperus metzii (Hochst. ex Steud.) Mattf. \& Kük. & Moro et al. 2014 & Hel & 1 & 1 & 0 & 0 & 0 \\
\hline & Cyperus obtusatus (J. Presl \& C. Presl) Mattf. \& Kük. & Matias et al. 2003; Moro et al. 2014 & Hel & 1 & 1 & 0 & 0 & 0 \\
\hline & Cyperus odoratus L. & Moro et al. 2014 & Hel & 0 & 1 & 1 & 0 & 0 \\
\hline & Cyperus polystachyos Rottb. & Matias et al. 2003; Moro et al. 2014; Ibiapina-Santos 2016 & Hel & 1 & 1 & 0 & 0 & 1 \\
\hline & Cyperus rotundus L. & Moro et al. 2014 & Hel & 1 & 0 & 0 & 0 & 0 \\
\hline & Cyperus sphacelatus Rottb. & Normando 2011 & $\mathrm{Hel}$ & 1 & 1 & 0 & 0 & 0 \\
\hline & Cyperus surinamensis Rottb. & Moro et al. 2014; Ibiapina-Santos 2016 & Hel & 1 & 1 & 0 & 0 & 0 \\
\hline & Cyperus uncinulatus Schrad. ex Nees & Moro et al. 2014 & Hel & 1 & 1 & 0 & 0 & 0 \\
\hline & Eleocharis acutangula (Roxb.) Schult. & Albuquerque et al. 2020 & $\mathrm{RE}$ & 1 & 0 & 0 & 0 & 0 \\
\hline & Eleocharis atropurpurea (Retz.) J.Presl \& C. Presl & Matias et al. 2003 & $\mathrm{RE}$ & 1 & 1 & 0 & 0 & 0 \\
\hline
\end{tabular}


Lígia Queiroz Matias, Felipe Martins Guedes,

Hugo Pereira do Nascimento and Júlia Caram Sfair

Table 1. Cont.

\begin{tabular}{|c|c|c|c|c|c|c|c|c|}
\hline Family & Species & Occurence indication & GF & PLE & TLE & PLO & TLO & ALE \\
\hline \multirow{28}{*}{ Cyperaceae } & Eleocharis elegans (Kunth) Roem. \& Schult. & Normando 2011 & RE & 0 & 1 & 0 & 0 & 0 \\
\hline & Eleocharis equisetoides (Elliott) Torr. & Moro et al. 2014 & $\mathrm{RE}$ & 1 & 0 & 0 & 0 & 0 \\
\hline & Eleocharis flavescens (Poir.) Urban & Ibiapina-Santos 2016 & $\mathrm{RE}$ & 0 & 1 & 0 & 0 & 0 \\
\hline & Eleocharis geniculata (L.) Roem. \& Schult. & Matias et al. 2003; Moro et al. 2014; Ibiapina-Santos 2016 & $\mathrm{RE}$ & 1 & 1 & 0 & 1 & 0 \\
\hline & Eleocharis interstincta (Vahl) Roem. \& Schult. & Tabosa et al. 2012; Ibiapina-Santos 2016; Albuquerque et al. 2020 & $\mathrm{RE}$ & 1 & 1 & 0 & 0 & 1 \\
\hline & Eleocharis maculosa (Vahl) Roem. \& Schult. & Normando 2011 & $\mathrm{RE}$ & 1 & 1 & 0 & 0 & 0 \\
\hline & Eleocharis mutata (L.) Roem. \& Schult. & Matias et al. 2003; Tabosa et al. 2012; Albuquerque et al. 2020 & $\mathrm{RE}$ & 1 & 1 & 0 & 0 & 0 \\
\hline & Eleocharis nana Kunth & Normando 2011 & $\mathrm{RE}$ & 1 & 0 & 0 & 0 & 0 \\
\hline & Eleocharis plicarhachis (Griseb.) Svenson & Matias et al. 2003 & $\mathrm{RE}$ & 1 & 1 & 0 & 0 & 0 \\
\hline & Eleocharis retroflexa (Poir.) Urban & Normando 2011 & $\mathrm{RE}$ & 0 & 1 & 0 & 0 & 0 \\
\hline & Eleocharis sellowiana Kunth & Matias et al. 2003 & $\mathrm{RE}$ & 1 & 0 & 0 & 0 & 0 \\
\hline & Eleocharis subfoliata C.B.Clarke & Normando 2011 & $\mathrm{RE}$ & 1 & 0 & 0 & 0 & 0 \\
\hline & Fimbristylis littoralis Gaudich. & Normando 2011 & Hel & 1 & 0 & 1 & 0 & 0 \\
\hline & Fimbrystilis cymosa $\mathrm{R}$. Br. & Matias et al. 2003; Moro et al. 2014 & Hel & 1 & 1 & 0 & 0 & 0 \\
\hline & Fimbrystilis dichotoma (L.) Vahl & Normando 2011 & Hel & 1 & 0 & 0 & 0 & 0 \\
\hline & Fimbristylis littoralis Gaudich. & Normando 2011 & Hel & 1 & 0 & 1 & 0 & 0 \\
\hline & Fimbrystilis vahlii (Lam.) Link & Normando 2011 & $\mathrm{Hel}$ & 1 & 0 & 0 & 0 & 0 \\
\hline & Fuirena robusta Kunth (*) & Moro et al. 2014 & Hel & 1 & 0 & 0 & 0 & 0 \\
\hline & Fuirena umbellata Rottb. & Matias et al. 2003 & Hel & 1 & 0 & 1 & 1 & 0 \\
\hline & Rhynchospora caracasana (Kunth) Boeckeler & Moro et al. 2014 & Hel & 1 & 0 & 0 & 0 & 0 \\
\hline & Rhynchospora contracta (Nees) J. Raynal & Normando 2011 & Hel & 1 & 0 & 0 & 0 & 0 \\
\hline & Rhynchospora exaltata Kunth & Moro et al. 2014 & Hel & 1 & 0 & 0 & 0 & 0 \\
\hline & Rhynchospora holoschoenoides (Rich.) Herter & Matias et al. 2003; Moro et al. 2014 & Hel & 1 & 1 & 0 & 0 & 0 \\
\hline & Rhynchospora riparia (Nees) Boeckeler & Matias et al. 2003 & Hel & 1 & 1 & 0 & 0 & 0 \\
\hline & Scleria bracteata Cav. & Normando 2011 & Hel & 0 & 0 & 1 & 0 & 0 \\
\hline & Scleria gaertneri Raddi & Guedes \& Nascimento 2016 & Hel & 0 & 0 & 1 & 0 & 0 \\
\hline & Scleria hirtella Sw. & Guedes \& Nascimento 2016 & Hel & 1 & 0 & 0 & 0 & 0 \\
\hline & Scleria macrophylla J. Presl \& C. Presl $1^{(*)}$ & Normando 2011 & Hel & 0 & 0 & 1 & 0 & 0 \\
\hline Dilleniaceae & Tetracera wildenowiana Steud. (") & Guedes \& Nascimento 2016 & Hel & 1 & 0 & 0 & 0 & 0 \\
\hline Euphorbiaceae & Caperonia palustris (L.) A. St.-Hil. ${ }^{\left({ }^{*}\right)}$ & Ibiapina-Santos 2016 & Hel & 1 & 1 & 0 & 0 & 1 \\
\hline \multirow{8}{*}{ Fabaceae } & Aeschynomene evenia C. Wright \& Sauvalle & Moro et al. 2014; Ibiapina-Santos 2016 & $\mathrm{RE}$ & 1 & 1 & 0 & 0 & 0 \\
\hline & Aeschynomene filosa Mart. & Tabosa et al. 2012; Ibiapina-Santos 2016 & RE & 0 & 1 & 0 & 0 & 0 \\
\hline & Aeschynomene rudis Benth. & Normando 2011 & $\mathrm{RE} / \mathrm{Hel}$ & 0 & 1 & 0 & 0 & 0 \\
\hline & Centrosema brasilianum (L.) Benth & Ibiapina-Santos 2016 & Hel & 0 & 1 & 0 & 0 & 1 \\
\hline & Centrosema plumieri (Tupin. ex Pers.) Benth & Ibiapina-Santos 2016 & Hel & 0 & 1 & 0 & 0 & 0 \\
\hline & Chamaecrista diphylla (L.) Greene & Guedes \& Nascimento 2016 & Hel & 1 & 1 & 0 & 0 & 0 \\
\hline & Chamaecrista ramosa var. ramosa (Vogel) H.S.Irwin \& Barneby & Guedes \& Nascimento 2016 & Hel & 1 & 1 & 0 & 0 & 0 \\
\hline & Desmodium triflorum (L.) DC. & Moro et al. 2014 & Hel & 1 & 0 & 0 & 0 & 0 \\
\hline
\end{tabular}


Table 1. Cont.

\begin{tabular}{|c|c|c|c|c|c|c|c|c|}
\hline Family & Species & Occurence indication & GF & PLE & TLE & PLO & TLO & ALE \\
\hline \multirow{15}{*}{ Fabaceae } & Discolobium hirtum Benth. & Tabosa et al. 2012 & Hel & 1 & 1 & 0 & 0 & 0 \\
\hline & Indigofera hirsuta $\mathrm{L}$. & Matias et al. 2003 & Hel & 1 & 1 & 0 & 0 & 0 \\
\hline & Indigofera microcarpa Desv. & Moro et al. 2014 & Hel & 1 & 0 & 0 & 0 & 0 \\
\hline & Macroptilium lathyroides (L.) Urban & Ibiapina-Santos 2016 & Hel & 0 & 1 & 0 & 0 & 0 \\
\hline & Mimosa camporum Benth. & Ibiapina-Santos 2016 & Hel & 0 & 1 & 0 & 0 & 0 \\
\hline & Mimosa candollei R. Grether & Ibiapina-Santos 2016 & $\mathrm{Hel}$ & 0 & 1 & 1 & 0 & 0 \\
\hline & Mimosa misera Benth. & Normando 2011 & Hel & 1 & 0 & 0 & 0 & 0 \\
\hline & Neptunia oleracea Lour. & $\begin{array}{l}\text { Tabosa et al. 2012; Moro et al. 2014; Paiva et al. 2014; } \\
\text { Ibiapina-Santos 2016; Albuquerque et al. } 2020\end{array}$ & RLF & 1 & 1 & 0 & 0 & 1 \\
\hline & Neptunia plena (L.) Benth. & Matias et al. 2003; Ibiapina-Santos 2016 & RLF & 1 & 1 & 1 & 0 & 1 \\
\hline & Parkinsonia aculeata L. & Normando 2011 & Hel & 1 & 1 & 0 & 0 & 0 \\
\hline & Senna alata (L.) Roxb. & Normando 2011 & Hel & 0 & 1 & 0 & 0 & 0 \\
\hline & Senna obtusifolia (L.) H.S. Irwin \& Barneby & Tabosa et al. 2012 & Hel & 0 & 1 & 0 & 0 & 0 \\
\hline & Sesbania exasperata Kunth & Ibiapina-Santos 2016 & Hel & 0 & 1 & 0 & 0 & 0 \\
\hline & Stylosanthes angustifolia Vogel & Matias et al. 2003; Moro et al. 2014 & Hel & 1 & 0 & 0 & 0 & 0 \\
\hline & Zornia latifolia Sm. & Matias et al. 2003 & $\mathrm{Hel}$ & 1 & 0 & 0 & 0 & 0 \\
\hline Gentianaceae & Schultesia guianensis (Aubl.) Malme & Matias et al. 2003 & $\mathrm{Hel}$ & 1 & 0 & 0 & 0 & 0 \\
\hline Heliotropiaceae & Heliotropium elongatum (Lehm.) I.M. Johnst. & Tabosa et al. 2012; Ibiapina-Santos 2016 & Hel & 0 & 1 & 0 & 0 & 0 \\
\hline \multirow{6}{*}{ Hydrocharitaceae } & Apalanthe granatensis (Bonpl.) Planch. & Tabosa et al. 2012; Ibiapina-Santos 2016 & RS & 1 & 1 & 0 & 0 & 1 \\
\hline & Egeria densa Planch. & Moro et al. 2014 & RS & 1 & 1 & 0 & 0 & 1 \\
\hline & Egeria najas Planch. & Matias et al. 2017 & RS & 1 & 1 & 0 & 0 & 1 \\
\hline & Najas arguta Kunth & Matias et al. 2017 & RS & 0 & 1 & 0 & 0 & 1 \\
\hline & Najas conferta (A.Braun) A.Braun & Tabosa et al. 2012; Ibiapina-Santos 2016 & RS & 1 & 1 & 0 & 0 & 1 \\
\hline & Najas marina L. & Matias et al. 2017 & RS & 1 & 0 & 0 & 0 & 0 \\
\hline Hydroleaceae & Hydrolea spinosa $\mathrm{L}$. & Moro et al. 2014; Ibiapina-Santos 2016 & $\mathrm{RE}$ & 1 & 1 & 1 & 1 & 1 \\
\hline \multirow{6}{*}{ Lentibulariaceae } & Utricularia cornuta Michx. & Guedes \& Matias 2020 & Hel & 1 & 0 & 0 & 0 & 0 \\
\hline & Utricularia erectiflora St.-Hil. \& Girard. & Guedes \& Matias 2020 & Hel & 1 & 1 & 0 & 0 & 0 \\
\hline & Utricularia foliosa L. & Tabosa et al. 2012; Ibiapina-Santos 2016; Guedes \& Matias 2020 & FS & 1 & 1 & 0 & 0 & 1 \\
\hline & Utricularia gibba L. & Moro et al. 2014; Ibiapina-Santos 2016; Guedes \& Matias 2020 & FS & 1 & 1 & 0 & 0 & 0 \\
\hline & Utricularia hydrocarpa Vahl. & Tabosa et al. 2012; Guedes \& Matias 2020 & FS & 0 & 1 & 0 & 0 & 0 \\
\hline & Utricularia trichophylla Spruce ex. Oliv. & Guedes \& Matias 2020 & Hel & 1 & 0 & 1 & 0 & 0 \\
\hline Loganiaceae & Spigelia anthelmia L. & Matias et al. 2003 & $\mathrm{RE}$ & 1 & 0 & 0 & 0 & 0 \\
\hline \multirow{2}{*}{ Lythraceae } & Ammannia latifolia $\mathrm{L}$. & Moro et al. 2014; Ibiapina-Santos 2016 & Hel & 1 & 1 & 1 & 0 & 0 \\
\hline & Cuphea campestres Mart. ex Koehne & Tabosa et al. 2012 & Hel & 0 & 1 & 0 & 0 & 0 \\
\hline \multirow{4}{*}{ Malvaceae } & Malachra radiata (L.) L. & Ibiapina-Santos 2016 & Hel & 1 & 1 & 0 & 0 & 0 \\
\hline & Melochia pyramidata L. & Ibiapina-Santos 2016 & Hel & 0 & 1 & 0 & 0 & 1 \\
\hline & Melochia tomentosa L. & Ibiapina-Santos 2016 & Hel & 1 & 1 & 1 & 0 & 1 \\
\hline & Waltheria indica $\mathrm{L}$. & Ibiapina-Santos 2016 & Hel & 0 & 1 & 0 & 0 & 0 \\
\hline
\end{tabular}


Lígia Queiroz Matias, Felipe Martins Guedes,

Hugo Pereira do Nascimento and Júlia Caram Sfair

Table 1. Cont

\begin{tabular}{|c|c|c|c|c|c|c|c|c|}
\hline Family & Species & Occurence indication & GF & PLE & TLE & PLO & TLO & ALE \\
\hline \multirow{2}{*}{ Marantaceae } & Thalia densibracteata Petersen & Ibiapina-Santos 2016 & $\mathrm{RE}$ & 0 & 1 & 0 & 0 & 0 \\
\hline & Thalia geniculata L. & Tabosa et al. 2012; Ibiapina-Santos 2016 & $\mathrm{RE}$ & 1 & 1 & 0 & 0 & 0 \\
\hline Melastomataceae & Pterolepis glomerata (Rottb.) Miq. & Moro et al. 2014 & $\mathrm{RE}$ & 1 & 0 & 0 & 0 & 0 \\
\hline Menyanthaceae & Nymphoides humboldtiana (Kunth) Kuntze & $\begin{array}{l}\text { Matias et al. 2003; Moro et al. 2014; Paiva et al. 2014; } \\
\quad \text { Ibiapina-Santos 2016; Albuquerque et al. } 2020\end{array}$ & RLF & 1 & 1 & 0 & 0 & 1 \\
\hline \multirow{9}{*}{ Nymphaeaceae } & Nymphaea amazonum Mart. \& Zucc. & Moro et al. 2014; Albuquerque et al. 2020 & RLF & 1 & 1 & 1 & 0 & 1 \\
\hline & Nymphaea ampla (Salisb.) DC. & Sousa \& Matias 2013 & RLF & 0 & 1 & 0 & 0 & 0 \\
\hline & Nymphaea jamesoniana Planch. & Ibiapina-Santos 2016 & RLF & 0 & 1 & 0 & 0 & 0 \\
\hline & Nymphaea lasiophylla Mart. \& Zucc. & $\begin{array}{l}\text { Matias et al. 2003; Tabosa et al. 2012; Moro et al. 2014; } \\
\text { Ibiapina-Santos 2016; Albuquerque et al. } 2020\end{array}$ & RLF & 1 & 1 & 1 & 0 & 1 \\
\hline & Nymphaea lingulata Wiersema & Ibiapina-Santos 2016; Albuquerque et al. 2020 & RLF & 1 & 1 & 0 & 0 & 1 \\
\hline & Nymphaea prolifera Wiersema & Sousa \& Matias 2013 & RLF & 1 & 0 & 0 & 0 & 0 \\
\hline & Nymphaea pulchella DC. & Ibiapina-Santos 2016; Albuquerque et al. 2020 & RLF & 1 & 1 & 1 & 0 & 0 \\
\hline & Nymphaea rudgeana G. Mey. & Matias et al. 2003; Moro et al. 2014 & RLF & 1 & 0 & 0 & 0 & 0 \\
\hline & Nymphaea tenerinervia Casp. & Moro et al. 2014 & RLF & 1 & 1 & 0 & 0 & 0 \\
\hline \multirow{5}{*}{ Onagraceae } & Ludwigia erecta (L.) H. Hara & Nascimento \& Matias (2021) & Hel & 1 & 1 & 0 & 0 & 0 \\
\hline & Ludwigia helminthorrhiza (Mart.) H. Hara & $\begin{array}{l}\text { Moro et al. 2014; Paiva et al. 2014; Ibiapina-Santos 2016; } \\
\text { Albuquerque et al. 2020; Nascimento \& Matias (2021) }\end{array}$ & RLF & 1 & 1 & 0 & 0 & 1 \\
\hline & Ludwigia hyssopifolia (G. Don) Exell & Moro et al. 2014; Ibiapina-Santos 2016; Nascimento \& Matias (2021) & $\mathrm{RE}$ & 1 & 1 & 1 & 1 & 1 \\
\hline & Ludwigia leptocarpa (Nutt.) H.Hara & Moro et al. 2014; Ibiapina-Santos 2016; Nascimento \& Matias (2021) & Hel & 1 & 1 & 1 & 0 & 1 \\
\hline & Ludwigia octovalvis (Jacq.) P.H.Raven & Matias et al. 2003; Nascimento \& Matias (2021) & Hel & 1 & 1 & 1 & 0 & 1 \\
\hline \multirow{8}{*}{ Plantaginaceae } & Angelonia biflora Benth. & Paiva et al. 2014 & Hel & 0 & 0 & 1 & 0 & 0 \\
\hline & Bacopa angulata (Benth.) Loefgr. \& Edwall & Moro et al. 2014 & $\mathrm{RE}$ & 1 & 0 & 0 & 0 & 0 \\
\hline & Bacopa aquatica Aubl. & Tabosa et al. 2012; Moro et al. 2014 & $\mathrm{RE}$ & 1 & 1 & 0 & 0 & 1 \\
\hline & Bacopa cochlearia (Huber) L.B.Sm. & Moro et al. 2014 & $\mathrm{RE}$ & 1 & 1 & 1 & 0 & 0 \\
\hline & Bacopa monnieri (L.) Wettst. & Moro et al. 2014 & $\mathrm{RE}$ & 1 & 0 & 1 & 0 & 0 \\
\hline & Scoparia dulcis L. & Paiva et al. 2014; Ibiapina-Santos 2016 & Hel & 1 & 1 & 0 & 1 & 0 \\
\hline & Stemodia foliosa Benth. & Guedes \& Nascimento 2016 & Hel & 1 & 0 & 0 & 0 & 1 \\
\hline & Stemodia maritima L. & Paiva et al. 2014; Ibiapina-Santos 2016 & Hel & 1 & 1 & 1 & 0 & 1 \\
\hline \multirow{10}{*}{ Poaceae } & Aristida adscensionis L. & Guedes \& Nascimento 2016 & Hel & 0 & 1 & 0 & 0 & 0 \\
\hline & Aristida amazonensis Longhi-Wagner (") & Guedes \& Nascimento 2016 & Hel & 1 & 0 & 0 & 0 & 0 \\
\hline & Cyphonanthus discrepans (Döll) Zuloaga \& Morrone $e^{(*)}$ & Normando 2011 & Hel & 1 & 0 & 0 & 0 & 0 \\
\hline & Digitaria ciliaris (Retz.) Koeler & Tabosa et al. 2012 & Hel & 0 & 1 & 0 & 0 & 0 \\
\hline & Digitaria horizontalis Willd. & Normando 2011 & Hel & 1 & 0 & 0 & 0 & 0 \\
\hline & Echinochloa colona (L.) Link & Tabosa et al. 2012; Ibiapina-Santos 2016 & Hel & 0 & 1 & 0 & 0 & 0 \\
\hline & Echinochloa polystachya (Kunth) Hitchc. & Tabosa et al. 2012; Paiva et al. 2014 & Hel & 1 & 1 & 0 & 0 & 1 \\
\hline & Eragrostis ciliaris (L.) R.Br. & Matias \& Nunes 2001 & Hel & 1 & 0 & 0 & 0 & 0 \\
\hline & Hymenachne amplexicaulis (Rudge) Nees & Ibiapina-Santos 2016 & $\mathrm{RE}$ & 1 & 1 & 0 & 0 & 1 \\
\hline & Panicum dichotomiflorum Michx. & Tabosa et al. 2012 & Hel & 0 & 1 & 0 & 0 & 0 \\
\hline
\end{tabular}


Table 1. Cont.

\begin{tabular}{|c|c|c|c|c|c|c|c|c|}
\hline Family & Species & Occurence indication & GF & PLE & TLE & PLO & TLO & ALE \\
\hline \multirow{11}{*}{ Poaceae } & Paspalidium geminatum (Forssk.) Stapf & Matias et al. 2003; Moro et al. 2014 & Hel & 1 & 1 & 0 & 0 & 0 \\
\hline & Paspalum densum Poir. & Normando 2011 & Hel & 1 & 0 & 0 & 0 & 1 \\
\hline & Paspalum distichum L." & Moro et al. 2014 & Hel & 1 & 0 & 0 & 0 & 0 \\
\hline & Paspalum orbiculatum Poir..$^{(*)}$ & Tabosa et al. 2012 & Hel & 0 & 1 & 0 & 0 & 0 \\
\hline & Paspalum repens P.J.Bergius & Moro et al. 2014; Ibiapina-Santos 2016 & Hel & 1 & 1 & 0 & 0 & 0 \\
\hline & Paspalum vaginatum $\mathrm{Sw}$. & Matias et al. 2003; Moro et al. 2014 & Hel & 1 & 0 & 0 & 0 & 0 \\
\hline & Reimarochloa brasiliensis (Spreng.) Hitch ${ }^{(*)}$ & Ibiapina-Santos 2016 & Hel & 1 & 1 & 0 & 0 & 0 \\
\hline & Rugoloa polygonata (Schrad.) Zuloaga & Normando 2011 & Hel & 1 & 0 & 0 & 0 & 1 \\
\hline & Sacciolepis myuros (Lam.) Chase (") & Normando 2011 & Hel & 1 & 0 & 0 & 0 & 1 \\
\hline & Sporobolus virginicus (L.) Kunth ${ }^{(*)}$ & Matias \& Nunes 2001 & Hel & 1 & 0 & 0 & 0 & 0 \\
\hline & $\begin{array}{c}\text { Urochloa arrecta (Hack. ex T.Durand \& Schinz) Morrone \& } \\
\text { Zuloaga }\end{array}$ & Ibiapina-Santos 2016 & Hel & 1 & 1 & 0 & 0 & 1 \\
\hline \multirow{2}{*}{ Podostemaceae } & Apinagia gardneriana Tul. & Guedes \& Nascimento 2016 & HAP & 0 & 0 & 1 & 0 & 0 \\
\hline & Mourera aspera (Bong.) Tul. () & Guedes \& Nascimento 2016 & HAP & 0 & 0 & 1 & 0 & 0 \\
\hline Polygalaceae & Polygala appendiculata Vell. & Moro et al. 2014 & Hel & 1 & 0 & 0 & 0 & 0 \\
\hline \multirow{2}{*}{ Polygonaceae } & Polygonum ferrugineum Wedd. & Moro et al. 2014; Paiva et al. 2014; Ibiapina-Santos 2016 & $\mathrm{RE}$ & 1 & 1 & 1 & 0 & 1 \\
\hline & Polygonum hispidum Kunth & Ibiapina-Santos 2016 & $\mathrm{RE}$ & 1 & 1 & 0 & 0 & 1 \\
\hline \multirow{10}{*}{ Pontederiaceae } & Eichhornia crassipes (Mart.) Solms & Paiva et al. 2014; Ibiapina-Santos 2016 & FE & 1 & 1 & 1 & 0 & 1 \\
\hline & Eichhornia diversifolia (Vahl) Urb. & Ibiapina-Santos 2016; Albuquerque et al. 2020 & RLF & 0 & 1 & 0 & 1 & 0 \\
\hline & Eichhornia heterosperma Alexander & Tabosa et al. 2012; Ibiapina-Santos 2016; Albuquerque et al. 2020 & $\mathrm{RE}$ & 0 & 1 & 0 & 0 & 0 \\
\hline & Eichhornia paniculata (Spreng.) Solms & Albuquerque et al. 2020 & $\mathrm{RE}$ & 0 & 1 & 0 & 0 & 1 \\
\hline & Eichhornia paradoxa (Mart. ex Schult. \& Schult.f.) Solms ${ }^{(*)}$ & Sousa et. al 2018 & $\mathrm{RE}$ & 0 & 1 & 0 & 0 & 0 \\
\hline & Heteranthera oblongifolia Mart. Ex Schult. \& Schult.f. & Tabosa et al. 2012; Ibiapina-Santos 2016 & $\mathrm{RE}$ & 1 & 1 & 0 & 0 & 0 \\
\hline & Heteranthera rotundifolia (Kunth) Griseb. & Sousa et al. 2018 & RLF & 0 & 1 & 0 & 0 & 0 \\
\hline & Heteranthera seubertiana Solms & Sousa et al. 2018 & $\mathrm{RE}$ & 0 & 1 & 0 & 0 & 0 \\
\hline & Hydrothrix gardneri Hook.f. & Tabosa et al. 2012 & RS & 1 & 0 & 0 & 0 & 1 \\
\hline & Pontederia parviflora Alexander & Ibiapina-Santos 2016 & $\mathrm{RE}$ & 1 & 1 & 0 & 0 & 0 \\
\hline Potamogetonaceae & Potamogeton pusillus $\mathrm{L}$. & Ibiapina-Santos 2016 & RS & 1 & 0 & 0 & 0 & 1 \\
\hline \multirow{3}{*}{ Rubiaceae } & Richardia grandiflora (Cham. \& Schlecht.) Steud. & Matias \& Nunes 2001 & Hel & 1 & 0 & 0 & 0 & 1 \\
\hline & Borreria scabiosoides Cham \& Schltdl. & Tabosa et al. 2012; Ibiapina-Santos 2016 & Hel & 0 & 1 & 0 & 0 & 0 \\
\hline & Borreria verticillata (L.) G.Mey. & Matias et al. 2003 & Hel & 1 & 0 & 0 & 0 & 0 \\
\hline Sphenocleaceae & Sphenoclea zeylanica Gaertn. & Ibiapina-Santos 2016 & Hel & 0 & 1 & 0 & 0 & 0 \\
\hline Turneraceae & Turnera weddelliana Urb. \& Rolfe (") & Moro et al. 2014 & Hel & 1 & 0 & 0 & 0 & 0 \\
\hline \multirow{2}{*}{ Typhaceae } & Typha angustifolia L. & Guedes \& Nascimento 2016 & $\mathrm{RE}$ & 1 & 0 & 0 & 0 & 0 \\
\hline & Typha domingensis Pers. & Ibiapina-Santos 2016 & $\mathrm{RE}$ & 1 & 1 & 0 & 0 & 0 \\
\hline \multirow{2}{*}{ Xyridaceae } & Xyris jupicai Rich. & Moro et al. 2014 & Hel & 1 & 0 & 0 & 0 & 0 \\
\hline & Xyris macrocephala Vahl & Matias et al. 2003; Moro et al. 2014 & $\mathrm{Hel}$ & 1 & 0 & 0 & 0 & 0 \\
\hline
\end{tabular}




\section{Discussion}

Our hypothesis that lentic ecosystems present more species of aquatic angiosperms was confirmed. However, our results show that the different aquatic ecosystems present distinct species composition and different proportions of sets of growth forms (bottom-rooted submerged, bottomrooted emergent, bottom-rooted emergent with floating leaves and/or stems, free-swimming submerged, freefloating emergent, haptophytes and helophytes) according to the ecosystem. This result does not corroborate the hypothesis that in permanent lentic ecosystems there would be a greater diversity of growth forms compared to the others.

Cyperaceae and Poaceae were the most representative families in the wetlands of Ceará state. These plant families also constitute the greatest richness of monocotyledons in the world (Bouchenak-Khelladi et al. 2014) and exhibit strong dominance in several wetlands worldwide (Sieben 2010; Rodríguez-Arias \& Benavides 2016; Oliveira et al.

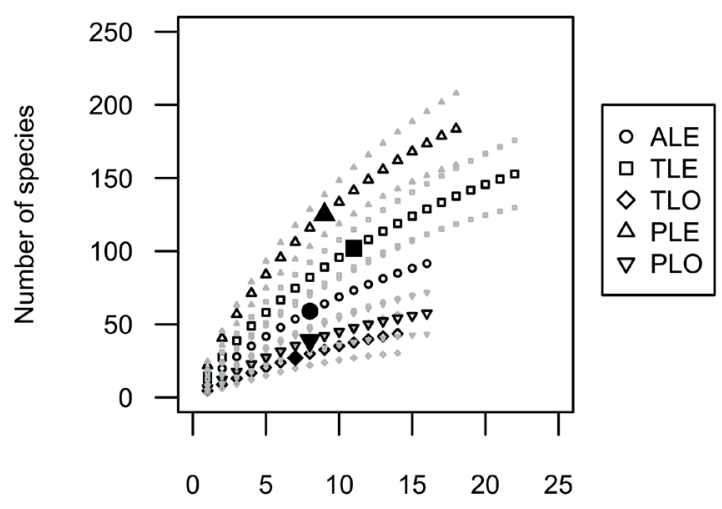

Number of wetlands

Figure 3. Rarefied and extrapolated (black symbols) number of species in the five ecosystems analysed. Confidence interval at $95 \%$ after 1000 randomizations (grey symbols, with the same shape of the correspondent rarefied and extrapolated number of species). The filled symbols are the observed number of species and have the same shape as the rarified and extrapolated number of species. The left side of the observed richness is the rarified and the right side is the extrapolated number of species. ALE = Artificial Lentic ecosystem; PLE = Permanent Lentic ecosystem; PLO = Permanent Lotic ecosystem; TLE $=$ Temporary Lentic ecosystem; TLO = Temporary Lotic ecosystem.
2019). Furthermore, the initial diversification of these plant groups occurred in the Paleogene (Bremer 2002), a period of intense rainfall and open landscapes (Cerling et al. 1998), probably in wetlands (Givnish et al. 2010). In addition, some Poales are restricted to aquatic ecosystems, such as Typhaceae, Xyridaceae and some genera of Eriocaulaceae (Bouchenak-Khelladi et al. 2014), which are also found in Ceará state.

Species of Cyperaceae, Poaceae, Fabaceae, Alismataceae and Malvaceae predominate in the wetland flora of Ceará and are mostly helophytes and bottom-rooted emergent hydrophytes. These growth forms stand out in lotic systems and the margins of lentic systems, habitats usually associated with primary succession. Notably, Cyperaceae and Poaceae species present efficient long-distance dispersal mechanisms and underground systems that allow for effective vegetative propagation (Goetghebeur 1998). Both families usually have the largest number of representatives in aquatic environments (Matias et al. 2003; Tabosa et al. 2012; Oliveira et al. 2019).

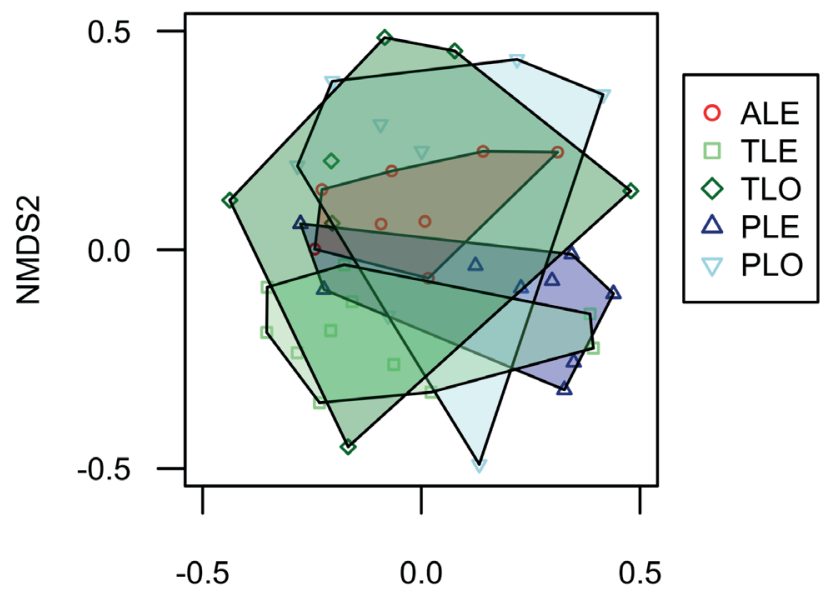

\section{NMDS1}

Figure 4. Nonmetric Multidimensional Scaling (NMDS) representing the grouping of the wetlands and their corresponding ecosystems. The PERMANOVA indicated the difference among the five ecosystems $\left(\mathrm{F}=1.25 ; \mathrm{R}^{2}=0.03 ; \mathrm{P}=0.03\right.$; Table 1$)$. $\mathrm{ALE}=$ Artificial Lentic ecosystem; PLE = Permanent Lentic ecosystem; $\mathrm{PLO}=$ Permanent Lotic ecosystem; TLE $=$ Temporary Lentic ecosystem; TLO = Temporary Lotic ecosystem. The stress of the NMDS is 0.29 .

Table 2. Permutational Multivariate Analysis of Variance Using Distance Matrices (PERMANOVA) results considering the five different habitats. The interaction is related the two factors: temporary/permanent/artifical and lotic/lentic. ALE = artificial lentic; $\mathrm{PLE}=$ permanent lentic; PLO = permanent lotic; TLE = temporary lentic; TLO = temporary lotic wetland.

\begin{tabular}{|c|c|c|c|c|c|c|}
\hline & DF & Sum of the squares & Mean of the squares & $\mathbf{F}$ & $\mathbf{R}^{2}$ & $\mathbf{P}$ \\
\hline Temporary (TLE, TLO)/ Permanent(PLE, PLO)/ Artificial (ALE) & 2 & 1.22 & 0.61 & 1.36 & 0.06 & 0.001 \\
\hline Lentic (ALE, PLE, TLE)/ Lotic (PLO, TLO) & 1 & 0.77 & 0.77 & 1.72 & 0.04 & 0.0002 \\
\hline Interaction & 1 & 0.56 & 0.56 & 1.26 & 0.03 & 0.039 \\
\hline Residuals & 38 & 16.99 & 0.44 & & 0.86 & \\
\hline Total & 42 & 19.54 & & & 1 & \\
\hline
\end{tabular}


The greatest species richness was found in permanent and temporary lentic ecosystems with the largest number of exclusive species (56.16\%). The stability of the water column contributed to species with different growth forms being able to colonize these ecosystems, resulting in local dominance of one or a few species and evident zonation patterns along environmental gradients from shoreline to limnetic zone border as a function of the variation in water depth (Spence 1982; McCreary 1991).

In the shallow coastal zone, the co-occurrence of helophytes and bottom-rooted hydrophytes constitute a stratified vegetation (Den Hartog \& Segal 1964), related to the typically strong competition among aquatic plant species (Gopal \& Goel 1993). And, as a greater number of species tend to co-occur at shallower depths in lentic systems of Ceará (Matias et al. 2003), helophytes and bottom-rooted emergent hydrophytes, represented by the Cyperaceae, Poaceae, Fabaceae, Alismataceae and Malvaceae families, predominate along the banks of the aquatic ecosystems of the state. In the limnetic region, assemblages tend to constitute strata of free-swimming submerged forms according to a gradient of light intensity as a function of
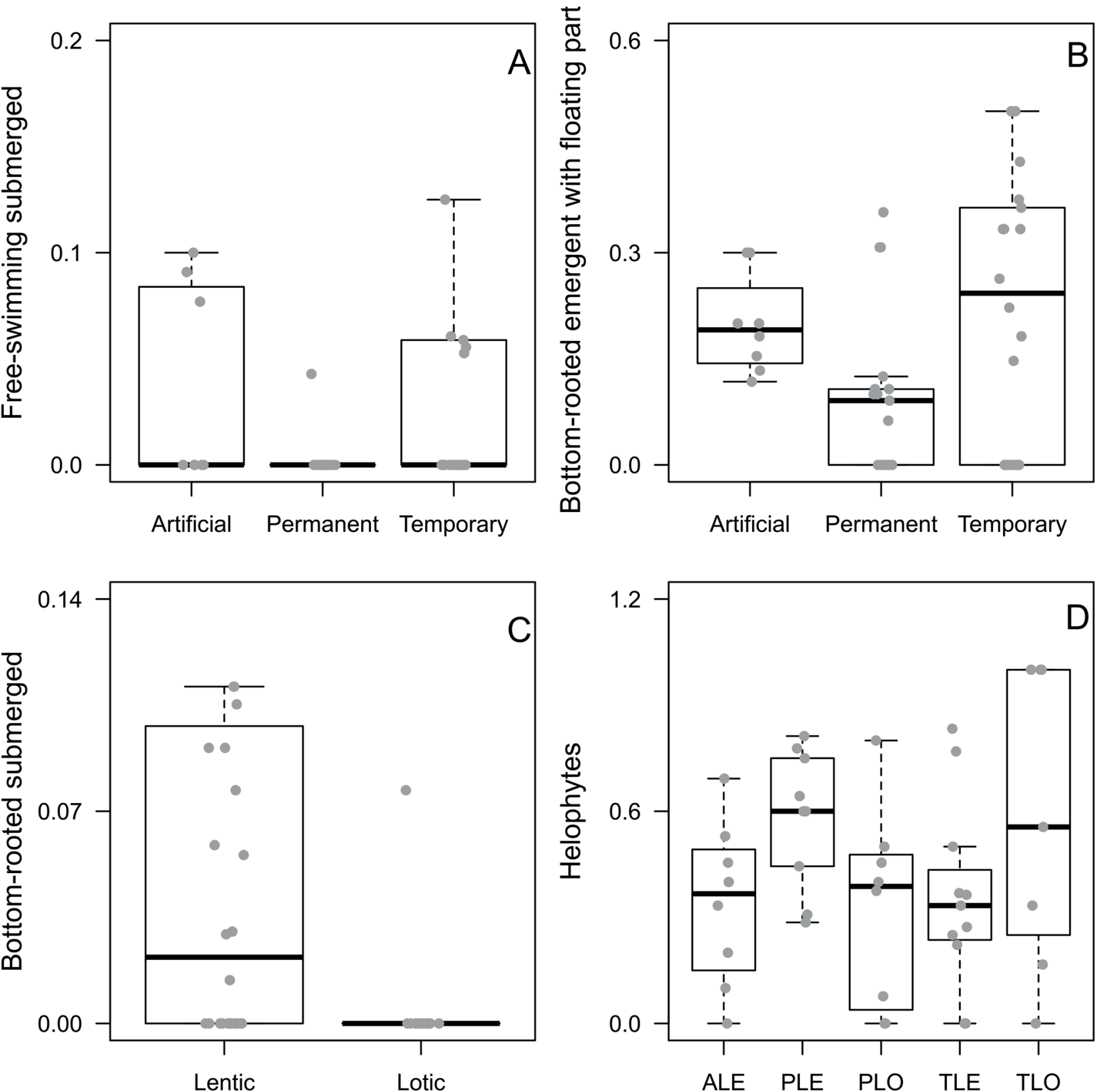

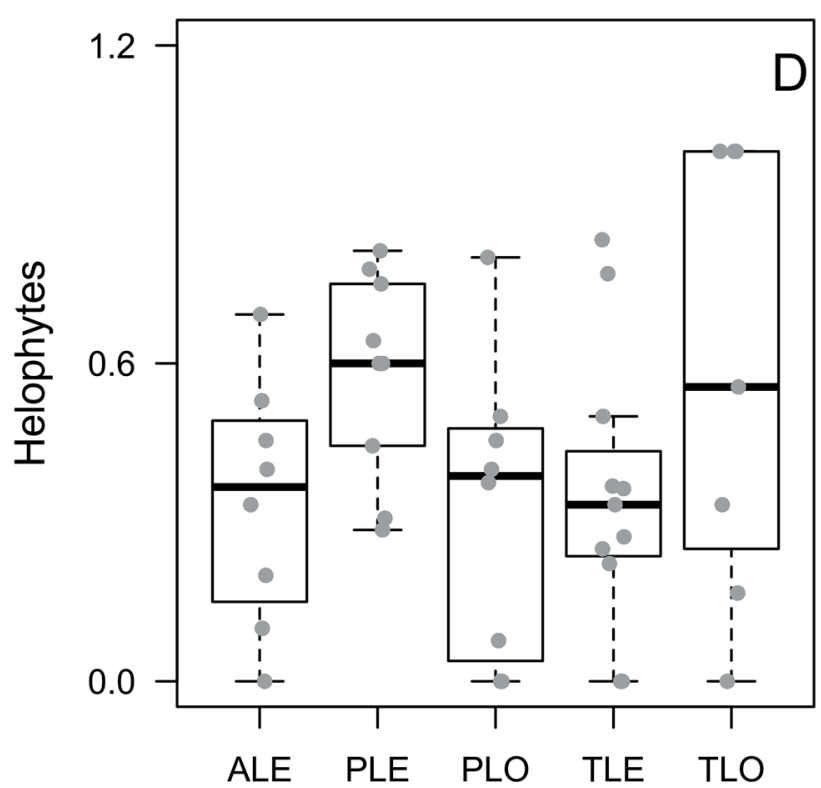

Figure 5. Boxplots showing the distribution of the proportion of growth forms among the aquatic ecosystems. A-B. Artificial, permanent, temporary; $\mathbf{C}$. lentic and lotic; D. different combinations of both. We show only the significant results in Table S2 in supplementary material. ALE = Artificial Lentic ecosystem; PLE = Permanent Lentic ecosystem; PLO = Permanent Lotic ecosystem; TLE = Temporary Lentic ecosystem; TLO = Temporary Lotic ecosystem. Grey points: the proportions of the life-form in the aquatic ecosystems. 
water depth (Rørslett \& Agami 1987) and occasionally, free-floating and bottom-rooted submerged forms in the extremes of the water column (Spence 1982).

The free-floating species showed no significant difference in the proportion of records among the aquatic ecosystems, but the proportion of free-swimming submerged and bottom-rooted emergents with floating leaves and/or stems was different between the artificial and natural lentic systems, and between natural lentic and lotic ones. Submerged plants interact intrinsically with the functioning of aquatic ecosystems, mobilizing nutrients and providing habitats for smaller dominant omnivore-planktivores (Meerhoff et al. 2003). Bottom-rooted emergents with floating leaves and/or stems can occupy the entire water column, especially in shallow lakes, resulting in the vanishing of submerged plants by accumulation of organic matter in the sediment and eutrophication of the environment due to the high production of floating leaf blades (Klok \& Velde 2017). Thus, these species assemblages affect, among other factors, the trophic levels of lentic ecosystems (Barko et al. 1986), resulting in a wide variation of growth forms.

The assemblages that compose the artificial aquatic systems include Utricularia foliosa and Ceratophyllum demersum, both free-swimming species. The shift in water flow allows submerged plants to form extensive vegetation banks that influence the dynamics of nutrients between water and sediment (Barbosa et al. 2020), constituting a common aspect in reservoirs of the Brazilian semiarid region. Other plants like Ludwigia helminthorrhiza, Neptunia oleracea, $N$. plena, Nymphaea amazonum, N. lasiophylla, N. lingulata and Nymphoides humboldtiana compose the assemblages of these systems as bottom-rooted emergents with floating leaves and/or stems. These species share a fast rate of vegetative reproduction, either by rhizomes or by stolons, so that in formations mixed with submerged macrophytes they present a pattern of relationship alternating between negative and positive interactions (Lycarião \& Dantas 2017). Additionally, they are generalists, except for $N$. ligulata (Sousa \& Matias 2013), and are found in most Brazilian regions (Flora do Brasil 2020 2020). Furthermore, there are fewer species in artificial systems than in natural lentic systems in Ceará state (14 or $6.39 \%$ are common species).

On the other hand, the proportion of bottom-rooted submerged forms was different between the lentic and lotic systems, with records of the following species limited to lentic ecosystems: Eriocaulon setaceum, Cabomba haynesii, Hydrothrix gardneri, Potamogeton pusillus and all Hydrocharitaceae species, with restricted occurrence of Najas marina in permanent lentic ecosystems. Species with this growth form were not recorded in lotic systems, probably due to their vulnerability to complete their life cycle due to abiotic factors such as water currents (Power et al. 2016).

Aquatic plant richness is related to other environmental factors in lentic systems (Alahuhta et al. 2014; Ferreira et al. 2015), which makes it difficult to generalize about the diversity of these ecosystems (Bubíková \& Hrivnák 2018). However, it is possible to observe that there is a set of exclusive species that are evolutionarily associated with these ecosystems. In this study, representatives of Nymphaeales (Cabombaceae, Nymphaeaceae), Monocotyledons (Alismataceae, Araceae, Hydrocharitaceae, Marantaceae, Potamogetonaceae, Pontederiaceae, Thyphaceae) and a few Eudicotyledons (Lentibulariaceae, Menyanthaceae, Hydroleaceae) have records limited to lentic ecosystems. Some of these families (Alismataceae, Araceae, Hydrocharitaceae, Lentibulariaceae, Pontederiaceae) have species with progressive adaptation to the aquatic environment, an evolutionary trend in monocots that is associated with vegetative differentiation more than in any other angiosperm group (Kremer \& Andel 1995).

In temporary lentic ecosystems, different growth forms colonize the entire water column in a space-time dynamic, i.e., these ecosystems have high species turnover (Tabosa et al. 2012). Thus, the species have not only overlapping niches, but are also phylogenetically related species and tend to compete among themselves (Ferreira et al. 2015). In these environments, few families did not have records, either because they are not associated with habitat specificity or because the populations are restricted to permanent lentic ecosystems (Araliaceae, Burmanniaceae, Linderniaceae, Potamogetonaceae, Xyridaceae), reflected by a considerable number of exclusive species (45 or $20.54 \%$ are exclusive to temporary lentic ecosystems). This shows that natural temporary lentic ecosystems contribute to the maintenance of aquatic plant richness in Ceará state, being recognized that natural ponds contributed most to regional biodiversity, supporting significantly many more species, more unique species and more rare species than other waterbody types (Williams et al. 2003).

The hydrophytes that have records restricted to these ecosystems were Eichhornia heterosperma, Eichhornia paradoxa, Heteranthera rotundifolia, Heteranthera seubertiana, Lemna minuta, Utricularia hydrocarpa, and the species endemic to northeastern Brazil, Echinodorus palaefolius and Echinodorus pubescens.

In contrast, the movement of the water column in lotic ecosystems is considered a significant driver of macrophytes diversity (Lacoul \& Freedman 2006; Bornette \& Puijalon 2011) because water speed selects species with morphological adaptations like streamlined shapes, straplike leaves or flat shoots (Chambers et al. 1991). Other adaptations include those that allow plants to attach firmly to the rocky substrate in rapid streams and waterfalls by specialized structures, such as disk-like holdfasts (haptera) found in Podostemaceae (Rutishauser et al. 2005), so that only Apinagia gardneriana and Mourera aspera have records limited to these ecosystems (Bubíková \& Hrivnák 2018). In the present study, lotic ecosystems showed low values of richness and a low number of exclusive species (12 or $5.47 \%)$. 
The rivers of the semiarid region present wide variation in the water column due to rainfall irregularity, selecting species with high resistance and resilience in response to hydric disturbances (Maltchick \& Pedro 2001). This may influence the low number of exclusive species, which tend to present mechanisms for survival in temporary environments based on ruderal strategies, due to the desiccation of the habitat and the consequent loss of biomass during the dry season (Albuquerque et al. 2020).

In temporary lotic systems, floods and droughts cause disturbances with different intensity, frequency and duration, determining the occurrence of aquatic communities. Therefore, species richness was lower in the puddles of the rivers and streams subject to flood events when compared to ponds (Pedro et al. 2006). This dynamic can explain the low richness found in the temporary rivers of Ceará, with the lowest number of exclusive species (8 or $3.65 \%)$.

Artificial lentic ecosystems showed species richness (86 spp.) close to the richness of lotic systems (83 spp.), and lower than natural lentic systems (359 spp.). Considering only the hydrophytes, the species that occupy these systems tend to be generalists (Echinodorus subalatus, Nymphaea amazonum, Neptunia plena, Ludwigia helminthorrhiza and L. leptocarpa) or species with predominant occurrence in natural lentic ecosystems with free-floating growth form (Lemna aequinoctialis, Pistia stratiotes, Spirodela intermedia, Wolffiella welwitschii), free-swimming submerged (Utricularia foliosa), bottom-rooted submerged (Cabomba haynesii, Apalanthe granatensis, Egeria densa, E. najas, Najas arguta, N. conferta, Potamogeton pusillus ) and bottom-rooted emergent forms (Echinodorus subalatus, Helanthium tenellum, Bacopa aquática, Stemodia foliosa, S. marítima, Polygonum ferrugineum and $P$. hispidum). These artificial systems exhibit irregular water level fluctuations related to the modifications of the reservoirs by human activity, being considered a special type of lentic environment (Hutchinson 1957), so that only a few species with free-floating forms have records from these environments in Ceará state: Eichhornia crassipes, Lemna aequinoctialis and Spirodela intermedia. The freefloating macrophytes tend to be limited by stream speed, being common in slow-flowing streams (Grinberga 2011), or when intercepted, tend to predominate in reservoirs (Paiva et al. 2014) mainly during the dry season (Lycarião \& Dantas 2017).

Among the species that occur in all five ecosystems, $P$. stratiotes has a pantropical distribution, while E. subalatus, $N$. amazonum, N. plena, L. helminthorrhiza and L. leptocarpa are of wide distribution in Brazil (Flora do Brasil 2020 2020) and in the American continent (Tropicos 2020). Some aquatic plants with wide distribution tend to present high levels of polymorphism and phenotypic plasticity related to environmental variables, allowing them to occur over a wide range of conditions (Lacoul \& Freedman 2006). Wide morphological variability in populations occurring in
Ceará has been described for E. subalatus (Matias 2007), N. amazonum (Sousa \& Matias 2013) and P. stratiotes (Andrade et al. 2013), which explains how these species tend to occupy different aquatic environments.

Helophytes predominated in the aquatic systems of Ceará state, accounting for $65 \%$ of the species. Vegetative reproduction is a predominant trait in this group, which may assure population maintenance at the ecological time scale (Li 2014). These species colonize the margins of aquatic systems forming assemblages that are affected by the dynamic of the substrate and the water (Deil et al. 2011). In addition to cryptophytic species, annual species occupy the margins of these systems, declining when the margins are little impacted by abiotic factors (e.g. waves) or when nutrients in substrate favour an increase in biomass and establishment of perennial rhizomatous helophytes (Hernández \& Rangel 2009).

An unusual finding in this study was that only the proportions of helophytic species differed in relation to the five types of aquatic systems. Among all the factors, the interactions between terrestrial and aquatic environments can explain the distribution and abundance of aquatic plants in many interaction scenarios (Lacoul \& Freedman 2006). Moreover, the organization of plant assemblages, regardless of the aquatic system type, may be influenced by biotic unpredictability, given that in arid regions ecological variability in life cycles is influenced by highly variable and unpredictable flow regimes and the impacts of land use and water resources (Choy et al. 2002).

The results showed that all waterbody types contributed to the macrophytes richness in the state, although lentic ecosystems have the highest richness values and exclusive species. So that the preservation of this flora must be considered not only for a specific type of aquatic ecosystem but for all possible types of freshwater habitats in Ceará state.

\section{Acknowledgements}

We acknowledge the Conselho Nacional de Desenvolvimento Científico e Tecnológico - CNPq for the fellowships granted to Hugo Pereira do Nascimento and Felipe Martins Guedes through the Programa Institucional de Bolsas de Iniciação Científica (PIBIC) of the Universidade Federal do Ceará.

\section{References}

Abell R, Thieme ML, Revenga C, et al. 2008. Freshwater ecoregions of the world: A new map of biogeographic units for freshwater biodiversity conservation. Bioscience 58: 403-414

Alahuhta J, Kaninen A, Hellsten S, et al. 2014. Variable response of functional macrophyte groups to lake characteristics, land use, and space: implications for bioassessment. Hydrobiologia 737: 201-214. 


\section{Lígia Queiroz Matias, Felipe Martins Guedes, Hugo Pereira do Nascimento and Júlia Caram Sfair}

Albuquerque AC, Rodrigues-Filho CAS, Matias LQ. 2020. Influence of climatic variables on CSR strategies of aquatic plants in a semiarid region. Hydrobiologia 847: 61-74.

Anderson MJ, Walsh DCI. 2013. PERMANOVA, ANOSIM, and the Mantel test in the face of heterogeneous dispersions: What null hypothesis are you testing? Ecological Monographs 83: 557-574.

Anderson MJ. 2001. A new method for non-parametric multivariate analysis of variance. Austral Ecology 26: 32-46.

Andrade IM, Mayo SJ, Silva MFS, Sousa DJL, Matias LQ, Ribeiro TA. 2013. The Araceae in Ceará, Brazil: humid forest plants in a semi-arid region. Rodriguésia 64: 445-477.

Barbosa VV, Severiano JS, Oliveira DA, Barbosa JEL. 2020. Influence of submerged macrophytes on phosphorus in a eutrophic reservoir in a semiarid region. Journal of Limnology 79: 138-150.

Barko JW, Adams MS, Clesceri NL. 1986. Environmental factors and their consideration in the management of submersed aquatic vegetation: a review. Journal of Aquatic Plant Management 24:1-10.

Bornette G, Puijalon S. 2011. Response of aquatic plants to abiotic factors: a review. Aquatic Sciences 73: 1-14.

Bouchenak-Khelladi Y, Muasya AM, Linder HP. 2014. A revised evolutionary history of Poales: origins and diversification Botanical Journal of the Linnean Society 175: 4-16.

Bremer K. 2002. Gondwanan evolution of the grass alliance of families (Poales). Evolution 56: 1374-1387.

Bubíková K, Hrivnák R. 2018. Relationships of macrophyte species richness and environment in different water body types in the Central European region Annales de Limnology-International Journal of Limnology 54: 35. doi: $10.1051 / \mathrm{limn} / 2018027$

Cerling TE, Ehleringer JR, Harris JM. 1998. Carbon dioxide starvation, the development of $\mathrm{C} 4$ ecosystems, and mammalian evolution. Philosophical Transactions of the Royal Society Series B 353: 159-171.

Chambers PA, Prepas EE, Hamilton HR, et al. 1991. Current velocity and its effect on aquatic macrophytes in flowing waters. Ecological Applications 1: 249-257.

Chao A, Gotelli NJ, Hsieh TC, et al. 2014. Rarefaction and extrapolation with Hill numbers: a framework for sampling and estimation in species diversity studies. Ecological Monographs 84: 45-67.

Choy SC, Thomson CB, Marshall JC. 2002. Ecological condition of central Australian arid-zone rivers. Water Science and Technology 45: 225-232.

Claudino-Sales V, Peulvast JP. 2002. Dune generation and ponds on the coast of Ceará State (Northeast Brazil). In: Allison RJ. (ed.) Applied Geomorphology. Chinchester, John Wiley \& Sons. p. 443-460.

Cook CDK. 1990. Aquatic Plant Book. Amsterdam, New York, SPB Academic Publishing.

Deil U, Freiburg IBr, Germany MA, et al. 2011.The vegetation of seasonal wetlands in extratropical and orotropical South America. Phytocoenologia 41: 1-34.

Den Hartog C, Segal S. 1964. A new classification of water-plant communities. Acta Botanica Neerlandica 13: 367-393.

Ferreira FS, Tabosa AB, Benvindo GR. 2015. Spatiotemporal ecological drivers of an aquatic plant community in a temporary tropical pool. Journal of Arid Environments 115: 66-72.

Flora do Brasil 2020. 2020. Jardim Botânico do Rio de Janeiro. http:// reflora.jbrj.gov.br/. 11 Mar. 2020.

Givnish TJ, Ames M, McNeal JR, et al. 2010. Assembling the tree of the monocotyledons: plastome sequence phylogeny and evolution of Poales. Annals of the Missouri Botanical Garden 97: 584-616.

Goetghebeur P. 1998. Cyperaceae. In: Kubitzki K. (ed.) The families and genera of vascular plants. Volume IV: Flowering Plants Monocotyledons: Alismatanae and Commelinanae (except Gramineae). Berlin, Heidelberg, Springer. p. 141-190.

Gopal B, Goel U. 1993. Competition and allelopathy in aquatic plant communities. Botanical Reviews 59: 155-210.

Grinberga L. 2011. Macrophyte species composition in streams of Latvia under different flow and substrate conditions. Estonian Journal of Ecology 60: 194-208.

Guedes FM, Nascimento HP, Matias LC. 2016. Ambientes aquáticos diferenciados agregam específicas comunidades de plantas aquáticas?
Revista Encontros Universitários da UFC. http://www.periodicos.ufc. br/eu/article/view/17346. 23 Mar. 2020.

Guedes FM, Matias LQ. 2020. Flora do Ceará, Brasil: Lentibulariaceae. Rodriguésia 70: e01892018. doi: 10.1590/2175-7860202071140

Hernández J, Rangel JO. 2009. La vegetación del humedal de Jaboque (Bogotá, D.C.). Caldasia 31: 355-379.

Hsieh TC, Ma KH, Chao A. 2013. iNEXT online: interpolation and extrapolation (Version 1.0) [Software]. http://chao.stat.nthu.edu. tw/blog/software-download/. 13 Mar. 2020.

Hutchinson GE. 1957. A Treatise on Limnology. New York, John Wiley \& Sons. Ibiapina-Santos L. 2016. Diversidade filogenética e fatores estruturantes de comunidades de plantas aquáticas em lagoas temporárias. MSc Thesis, Universidade Federal do Ceará, Fortaleza.

Junk W, Piedade MTF, Lourival R, et al. 2014. Brazilian wetlands: Definition, delineation and classification for research, sustainable management and protection. Aquatic Conservation: Marine and Freshwater Ecosystems 24: 5-22.

Klok PF, Velde G. 2017. Plant traits and environment: floating leaf blade production and turnover of Waterlilies. Peer J-the Journal of Life and Environmental Sciences 5: e3212. doi: 0.7717/peerj.3212

Koehler S, Bove CP. 2004. Alismatales from the Araguaia River Basin (MT/ GO, Brazil). Brazilian Journal of Botany 27: 439-452.

Kremer P, Andel J. 1995. Evolutionary aspects of life forms in angiosperm families. Acta Botanica Neerandica 44: 469-479

Lacoul P, Freedman B. 2006. Environmental influences on aquatic plants in freshwater ecosystems. Environmental Reviews 14: 89-136

Li W. 2014. Environmental opportunities and constraints in the reproduction and dispersal of aquatic plants. Aquatic Botany 118: 62-70.

Lycarião TA, Dantas EW. 2017. Interactions between different biological forms of aquatic macrophytes in a eutrophic tropical reservoir in Northeastern Brazil. Revista de Biologia Tropical 65: 1095-1104.

MacKay W. 1991. The role of ants and termites in desert communities. In: Polis G. (ed.) The ecology of desert communities. Tucson, The University of Arizona Press. p. 113-150.

Maltchick L, Bianchini I. 2006. Hydrologic cycle and dynamics of aquatic macrophytes in two intermittent rivers of the semi-arid region of Brazil. Brazilian Journal of Biology 66: 575-585.

Maltchick L, Pedro F. 2001. Responses of Aquatic Macrophytes to Disturbance by Flash Floods in a Brazilian Semiarid Intermittent Stream. Biotropica 33: 566-572.

Maltchick L, Costa MAJ, Duarte MDC. 1999. Inventory of Brazilian semi-arid shallow lakes. Anais da Academia Brasileira de Ciências 71: 801-808.

Maltchick L, Medeiros ESF. 2006. Conservation importance of semiarid streams in north-eastern Brazil: implications of hydrological disturbance and species diversity. Aquatic Conservation: Marine and Freshwater Ecosystems 16: 665-677.

Mangiafico S. 2019. rcompanion: functions to support extension education program evaluation. R package version 2.2.1. https://CRAN.R-project. org/package=rcompanion. 04 Mar. 2020.

Matias LQ, Amado RE, Nunes E. 2003. Macrófitas aquáticas da lagoa de Jijoca de Jericoacoara, Ceará, Brasil. Acta Botanica Brasilica 17: 623-631.

Matias LQ, Gonzalez HHS, Oliveria WR. 2017. Flora do Ceará: Hydrocharitaceae e as fanerógamas marinhas: Cymodoceaceae, Ruppiaceae. Rodriguésia 68: 1333-1346.

Matias LQ, Nunes E. 2001. Levantamento florística da Área de proteção Ambiental de Jericoacoara, Ceará, Brasil. Acta Botanica Brasilica 15: 35-43.

Matias LQ, Sousa DJL. 2011. Alismataceae no estado do Ceará, Brasil. Rodriguésia 62: 887-900.

Matias LQ. 2007. O gênero Echinodorus (Alismataceae) no domínio da Caatinga brasileira. Rodriguésia 62: 887-900.

McArdle BH, Anderson MJ. 2001. Fitting multivariate models to community data: a comment on distance-based redundancy analysis. Ecology 82: 290-297.

McCreary NJ. 1991. Competition as a mechanism of submersed macrophyte community structure. Aquatic Botany 41:177-193.

McNeely J. 2003. Biodiversity in arid regions: Values and perceptions. Journal of Arid Environments 54: 61-70. 


\section{Breaking the misconception of a dry and lifeless semiarid region: the diversity and distribution of aquatic flora in wetlands of the Brazilian Northeast}

Medeiros ESF, Maltchick L. 1999. The effects of hydrological disturbance on the intensity of infestation of Lernaea cyprinacea in an intermittent stream fish community. Journal of Arid Environments 43: 351-356.

Meerhoff M, Mazzeo N, Moss B, Rodríguez-Gallego L. 2003. The structuring role of free-floating versus submerged plants in a subtropical shallow lake. Aquatic Ecology 37: 377-391.

Moro MF, Sousa DJL, Matias LQ. 2014. Rarefaction, richness estimation and extrapolation methods in the evaluation of unseen plant diversity in aquatic ecosystems. Aquatic Botany 117: 48-55.

Moro MF, Souza VC, Oliveira-Filho AT, et al. 2012. Alienígenas na sala: o que fazer com espécies exóticas em trabalhos de taxonomia florística e fitossociologia? Acta Botanica Brasilica 26: 991-999.

Nascimento HP, Matias LQ. 2021. Flora do Ceará, Brasil: Onagraceae. Rodriguésia 72: e01732019. doi: 10.1590/2175-7860202172029

Normando LRO. 2011. Fatores espaço-temporais e riqueza de macrófitas aquáticas de lagoas temporárias do semiárido do Brasil. MSc Thesis, Universidade Federal do Ceará, Fortaleza.

Ogle DH, Wheeler P, Dinno A. 2020. FSA: fisheries stock analysis. R package version 0.8.30. https://github.com/droglenc/FSA. 20 May 2020.

Oksanen J, Blanchet FG, Friendly M, et al. 2019. Vegan: community ecology package. R package version 2.5-6. https://CRAN.R-project. org/package=vegan. 10 May 2020.

Oliveira LS, Andrade BO, Boldrini II, Moço CC. 2019. Aquatic vascular plants of South Brazil: checklist and a comparative floristic approach. Acta Botanica Brasilica 3: 709-715.

Paiva JRA, Matias LQ, Martins FR, Becker H. 2014. Does distance between aquatic plant assemblages matter in defining similarity between them during high water-level periods. Lakes and Reservoirs: Research and Management 19: 37-45.

Pedro F, Maltchick L, Biachini Jr I. 2006. Hydrologic cycle and dynamics of aquatic macrophytes in two intermittent rivers of the semi-arid region of Brazil. Brazilian Journal of Biology 66: 575-585.

Power ME, Stout RJ, Cushing CE, et al. 2016. Biotic and Abiotic Controls in River and Stream Communities. Journal of the North American Benthological Society 7: 456-479.

R Development Core Team. 2020. R: A language and environment for statistical computing. R Foundation for Statistical Computing, Vienna, Austria. https://www.R-project.org/. 25 May 2020.
Rodríguez-Arias CE, Benavides AMS. 2016. Vegetación acuática de los humedales de la microcuenca alta de la quebrada Estero, San Ramón de Alajuela, Costa Rica. Brenesia 85/86: 9-20.

Rørslett B, Agami M.1987. Downslope limits of aquatic macrophytes: a test of the transient niche hypothesis. Aquatic Botany 29: 83-95.

Rutishauser R, Pfeifer E, Alejandro Novelo R, et al. 2005. Diamantina lombardii - an odd Brazilian member of the Podostemaceae. FloraMorphology, Distribution, Functional Ecology of Plants 200: 245-255.

Sculthorpe CD. 1967. The biology of aquatic plants. London, Edward Arnold.

Sieben EJ, Morris CD, Kotze DC, et al. 2010. Changes in plant form and function across altitudinal and wetness gradients in the wetlands of the Maloti-Drakensberg, South Africa. Plant Ecology 207: 107-119.

Silva IC, Bove CP, Koschnistzche C. 2015. Plantas de corredeiras: reprodução e conservação de Podostemaceae. Natureza on Line (Espírito Santo) 13: 6-11.

Silva LAC, Araujo RCP, Maia LP, et al. 2007. Zoneamento ecológicoeconômico da zona costeira do Estado do Ceará. Annais do XLV Congresso da Sociedade Brasileira de Sociologia, Administração e Economia Rural. Londrina, SOBER. p. 1-20.

Sokal RR, Rohlf FJ. 1995. Biometry. 3rd. edn. New York, W. H. Freeman and Company.

Sousa DJL, Campelo MJA, Matias LQ. 2018. Flora do Ceará: Pontederiaceae. Rodriguésia 69: 1641-1657.

Sousa DJL, Matias LQ. 2013. A família Nymphaeaceae no Estado do Ceará. Rodriguésia 64: 049-059.

Spence DHN. 1982. The zonation of plants in freshwater lakes. Advances in Ecological Research 12: 37-126.

Tabosa AB, Matias LQ, Martins FR. 2012. Live fast end die young: The aquatic macrophyte dynamics in a temporary pool in the Brazilian semiarid region. Aquatic Botany 102: 71-78.

Tropicos ${ }^{\circ}$ - The tropics database. 2020. Missouri Botanical Garden. http:// www.tropicos.org. 23 Apr. 2020.

Wetzel RG. 2001. Limnology. San Diego, Academic Press.

Williams P, Whitfield M, Biggs J, et al. 2003. Comparative biodiversity of rivers, streams, ditches and ponds in an agricultural landscape in Southern England. Biological Conservation 115: 329-341. 